\title{
Normal Products in the Thirring Model*
}

\author{
JOHN H. LOWENSTEIN \\ Departamento de Física, Universidade de São Paulo \\ São Paulo, Brasil
}

Received December 9, 1969

\begin{abstract}
The short-distance behavior of products of fields is studied in the Thirring model of a self-interacting, massless, spinor field in two dimensions. One is able to construct normal products which share most of the convenient properties of the free-field Wick products. A modified form of Wilson's expansion formula is verified. The original formulation of Wilson's hypothesis is seen to hold to every finite order in the coupling constant, but fails in the exact solution of the model for sufficiently strong coupling.
\end{abstract}

\section{Introduction}

In relativistic quantum field theory, quantities of physical interest (e.g. currents, Lagrangian densities) are often defined formally in terms of products of field operators at a single point. It is thus of considerable importance to obtain greater mathematical respectability for such objects. In particular, one would like to formulate precisely the transition from the product,

$$
A^{(1)}\left(x_{1}\right) A^{(2)}\left(x_{2}\right) \ldots A^{(n)}\left(x_{n}\right)
$$

defined as an $n$-fold operator-valued distribution, to a local, covariant operator-valued distribution in a single variable (normal product),

$$
N\left[A^{(1)} A^{(2)} \ldots A^{(n)}\right](x) .
$$

A systematic framework for studying the singularity structure of products of field operators has been proposed by Wilson [4], who claims that every product of the type (1.1) should have, for sufficiently small $x_{i}-x$, an expansion of the form

$$
A^{(1)}\left(x_{1}\right) A^{(2)}\left(x_{2}\right) \ldots A^{(n)}\left(x_{n}\right)=\sum_{k} h^{(k)}\left(x_{1}, x_{2}, \ldots, x_{n}\right) \chi^{(k)}(x)+r\left(x_{1}, \ldots, x_{n}\right)
$$

where the $h^{(k)}\left(x_{1}, x_{2}, \ldots, x_{n}\right)$ are complex-valued, possibly singular functions, the $\chi^{(k)}(x)$ are local, covariant fields, and $r\left(x_{1}, x_{2}, \ldots x_{n}\right)$ vanishes for $x_{i}=x$, all $i$. The $\chi^{(k)}(x)$ all may be interpreted as normal

* Research supported by the Banco Nacional do Desenvolvimento Econômico and the Conselho Nacional de Pesquisas. 
products ("generalized Wick products") of the basic fields of the theory and their derivatives. Moreover, the dominant singularities of the $h^{(k)}\left(x_{1}, x_{2}, \ldots, x_{n}\right)$ are assumed to be essentially independent of the mass parameters of the theory, so that their short-distance behavior may be determined by dimensional arguments alone. Wilson [4] and Brandt [5] have verified the above hypothesis in specific cases of renormalized Lagrangian perturbation theory. In the examples treated the coefficient functions $h^{(k)}\left(x_{1}, x_{2}, \ldots, x_{n}\right)$ have a natural interpretation as renormalization counter terms.

It is not known to what extent Wilson's expansion is generally valid. Moreover, it may in fact be possible to obtain a more concise description of the short-distance behavior of quantum fields. For example, in the case of the free scalar field a more economical formalism is available, namely, the well known Wick-product expansion [1]:

$$
\begin{aligned}
& A\left(x_{1}\right) A\left(x_{2}\right) \ldots A\left(x_{n}\right) \\
& =\sum_{\substack{S \\
S \cup T=\{1,2, \ldots, n\} \\
S \cap T=\emptyset}}\left\langle 0\left|A\left(x_{S 1}\right) A\left(x_{S 2}\right) \ldots A\left(x_{S m_{S}}\right)\right| 0\right\rangle: A\left(x_{T 1}\right) \ldots A\left(x_{T n_{T}}\right):
\end{aligned}
$$

To arrive at Wilson's expansion for this product, one makes a Taylor expansion of the Wick products about the point $x$. To what extent the Wick products can be generalized, to what we shall call normal products, in less trivial field theories is far from clear.

It is the purpose of the present article to investigate the short-distance behavior of products of fields in a particular soluble model, the Thirring model [2] of a massless spinor field in a space-time of two dimensions, in order to test the applicability of Wilson's conjecture in a non-perturbative context, as well as to explore the possibility of a normalproduct expansion in this case. We shall discover that Wilson's hypothesis is valid to every order in perturbation theory, but fails for the exact solution of the model for sufficiently large coupling constants. However, modifying the hypothesis slightly, we shall be able to obtain formulas for the current (in Section IV) and for the equation of motion (in Section V) which are strictly analogous to those derived by Wilson and Brandt in renormalized perturbation theory.

\section{The Thirring Model}

The self-interacting, massless, spinor field in a space-time of two dimensions (Thirring model) [2] has been formulated in a mathematically satisfactory way by Klaiber [3] (see the bibliography of [3] for references to prior work). We now give an outline of Klaiber's construction. 
a) Definition of the Free, Massless, Spinor Field

$$
\psi_{i}(x)=\frac{1}{\sqrt{2 \pi}} \int d p^{1}\left[a^{*}\left(p^{1}\right) e^{i p x}+b\left(p^{1}\right) e^{-i p x}\right] u_{i}\left(p^{1}\right), \quad p^{0}=\left|p^{1}\right|, i=1,2
$$

where

The field $\psi$ satisfies

$$
\begin{aligned}
\left\{a\left(p^{1}\right), a^{*}\left(q^{1}\right)\right\} & =\left\{b\left(p^{1}\right), b^{*}\left(q^{1}\right)\right\}=\delta\left(p^{1}-q^{1}\right), \\
\left\{a\left(p^{1}\right), a\left(q^{1}\right)\right\} & =\left\{a\left(p^{1}\right), b^{*}\left(q^{1}\right)\right\}=0, \\
\left\{b\left(p^{1}\right), b\left(q^{1}\right)\right\} & =\left\{a\left(p^{1}\right), b\left(q^{1}\right)\right\}=0, \\
u\left(p^{1}\right) & =\left(\begin{array}{l}
\theta\left(-p^{1}\right) \\
\theta\left(p^{1}\right)
\end{array}\right) .
\end{aligned}
$$

with

$$
\gamma^{\mu} \partial_{\mu} \psi(x)=0, \quad\{\psi(x), \bar{\psi}(y)\}=\frac{1}{i} S(x-y),
$$

$$
\begin{gathered}
\gamma^{0}=\left(\begin{array}{ll}
0 & 1 \\
1 & 0
\end{array}\right), \quad \gamma^{1}=\left(\begin{array}{rr}
0 & 1 \\
-1 & 0
\end{array}\right), \quad \gamma^{5}=\gamma^{0} \gamma^{1}=\left(\begin{array}{rr}
-1 & 0 \\
0 & 1
\end{array}\right), \\
S(\xi)=i\left(\begin{array}{cc}
0 & \delta\left(\xi^{0}+\xi^{1}\right) \\
\delta\left(\xi^{0}-\xi^{1}\right) & 0
\end{array}\right) .
\end{gathered}
$$

b) Definition of the Conserved Current and Pseudocurrent

$$
\begin{aligned}
j_{\mu}(x) & =: \bar{\psi}(x) \gamma_{\mu} \psi(x):, \\
\tilde{j}_{\mu}(x) & =: \bar{\psi}(x) \gamma_{\mu} \gamma^{5} \psi(x):=\varepsilon_{\mu v} j^{\nu}(x), \quad \varepsilon=\left(\begin{array}{rr}
0 & 1 \\
-1 & 0
\end{array}\right) \\
\partial^{\mu} j_{\mu} & =0=\partial^{\mu} \tilde{j}_{\mu}, \\
\square j_{\mu} & =0=\square \tilde{j_{\mu}} .
\end{aligned}
$$

Conserved charge and pseudocharge in the free theory may then be defined as

$$
Q=\int_{x^{0}=t} d x^{1} j_{0}(x), \quad \tilde{Q}=\int_{x^{0}=t} d x^{1} \tilde{j}_{0}(x) .
$$

c) Current Potentials with Infrared Cut-offs

$$
\begin{aligned}
j^{-}(x)= & \frac{1}{\sqrt{2 \pi}} \int \frac{d k^{1}}{\sqrt{2 k^{0}}} c\left(k^{1}\right)\left(e^{-i k x}-\theta\left(\varkappa-k^{0}\right)\right), \quad k^{0}=\left|k^{1}\right|, \\
\tilde{j}^{-}(x)= & \frac{1}{\sqrt{2 \pi}} \int \frac{d k^{1}}{\sqrt{2 k^{0}}} \varepsilon\left(k^{1}\right) c\left(k^{1}\right)\left(e^{-i k x}-\theta\left(x-k^{0}\right)\right), \quad \varepsilon\left(k^{\prime}\right)=\frac{k^{1}}{\left|k^{1}\right|}, \\
j^{+}(x)= & \left(j^{-}(x)\right)^{\dagger}, \quad \tilde{j}^{+}(x)=\left(\tilde{j}^{-}(x)\right)^{\dagger}, \\
c\left(k^{1}\right)= & \frac{i}{\sqrt{k^{0}}} \int d p^{1}\left\{\theta\left(k^{1} p^{1}\right)\left[b^{*}\left(p^{1}\right) b\left(p^{1}+k^{1}\right)-a^{*}\left(p^{1}\right) a\left(p^{1}+k^{1}\right)\right]\right. \\
& \left.+\theta\left(p^{1}\left(k^{1}-p^{1}\right)\right) a\left(k^{1}-p^{1}\right) b\left(p^{1}\right)\right\} .
\end{aligned}
$$


Here $x$ is an arbitrary positive cut-off parameter with dimension of energy. One verifies

$$
\begin{aligned}
& j_{\mu}(x)=\frac{1}{\sqrt{\pi}} \partial_{\mu}\left(j^{-}(x)+j^{+}(x)\right)=\frac{1}{\sqrt{\pi}} \partial_{\mu} j(x), \\
& \tilde{j}_{\mu}(x)=\frac{1}{\sqrt{\pi}} \partial_{\mu}\left(\tilde{j}^{-}(x)+\tilde{j}^{+}(x)\right)=\frac{1}{\sqrt{\pi}} \partial_{\mu} \tilde{j}(x) .
\end{aligned}
$$

\section{d) Commutation Relations}

$$
\begin{aligned}
{\left[j^{-}(x), j^{+}(y)\right] } & =\left[\tilde{j}^{-}(x), \tilde{j}^{+}(y)\right]=\frac{1}{i} D_{\varkappa x}^{-}(x, y), \\
{\left[j^{-}(x), \tilde{j}^{+}(y)\right] } & =\left[\tilde{j}^{-}(x), j^{+}(y)\right]=\frac{1}{i} \tilde{D}_{\varkappa \varkappa}^{-}(x, y), \\
{\left[j^{ \pm}(x), \psi(y)\right] } & =-\sqrt{\pi}\left[D_{\varkappa}^{ \pm}(x, y)+\gamma^{5} \tilde{D}_{\varkappa}^{ \pm}(x, y)\right] \psi(y), \\
{\left[\tilde{j}^{ \pm}(x), \psi(y)\right] } & =-\sqrt{\pi}\left[\tilde{D}_{\varkappa}^{ \pm}(x, y)+\gamma^{5} D_{\varkappa}^{ \pm}(x, y)\right] \psi(y), \\
{[Q, \Psi(x)] } & =-\psi(x), \\
{[\tilde{Q}, \psi(x)] } & =-\gamma^{5} \psi(x), \\
D_{\varkappa \varkappa}^{ \pm}(x, y) & =D^{ \pm}(x-y)-\Delta^{ \pm}(x)+\Delta^{\mp}(y), \\
D_{\varkappa}^{ \pm}(x, y) & =D^{ \pm}(x-y)+\Delta^{\mp}(y), \\
D^{ \pm}(\xi) & =\mp \frac{i}{2 \pi} \int \frac{d p^{1}}{2 p^{0}}\left(e^{ \pm i p \xi}-\theta\left(\varkappa-p^{0}\right)\right) \\
& =\mp \frac{1}{4 \pi i} \log \left(-\mu^{1} \xi^{2} \mp i \varepsilon \xi^{0}\right), \quad \mu=e^{-\Gamma^{\prime}(1)} \varkappa, \\
\Delta^{ \pm}(x) & = \pm \frac{i}{k^{0}} \int \frac{d k^{1}}{k^{0}} \theta\left(\varkappa-k^{0}\right)\left(e^{\mp i k x}-1\right) .
\end{aligned}
$$

The analogous expressions for $\tilde{D}_{\varkappa x}^{ \pm}(x, y), \tilde{D}_{\varkappa}^{ \pm}(x, y), \tilde{D}^{ \pm}(\xi), \tilde{\Delta}^{ \pm}(x)$ are obtained by replacing $\int \frac{d p^{1}}{p^{0}}$ by $\int \frac{d p^{1}}{p^{1}}=\int \frac{d p^{1}}{p^{0}} \varepsilon\left(p^{1}\right)$ in the above formulas.

where

\section{e) Definition of the Interacting Field}

$$
\phi(x)=e^{i \chi^{+}(x)} \psi(x) e^{i x^{-}(x)}
$$

$$
\begin{aligned}
\chi^{ \pm}(x)= & \alpha j^{ \pm}(x)+\beta \gamma^{5} \tilde{j}^{ \pm}(x)+(\sqrt{\pi}-\alpha)\left[\alpha Q \Delta^{ \pm}(x)+\beta \tilde{Q} \tilde{\Delta}^{ \pm}(x)\right] \\
& +\gamma^{5}(\sqrt{\pi}-\beta)\left[\alpha Q \tilde{\Delta}^{ \pm}(x)+\beta \tilde{Q} \Delta^{ \pm}(x)\right] .
\end{aligned}
$$


The parameters $\alpha$ and $\beta$ may be fixed by specifying the coupling constant, $g=\sqrt{\pi}(\alpha-\beta)$, and requiring that $\phi$ transform as a spin $-\frac{1}{2}$ field with the usual anticommutation relations for spacelike separations (this implies $\alpha \beta-\alpha \sqrt{\pi}-\beta \sqrt{\pi}=0$ ). The field thus defined is an operator-valued distribution, with domain $\mathscr{D}_{\phi}$ generated by acting on the vacuum state with polynomials in $\phi$ and $\phi^{\dagger}$. Inhomogeneous Lorentz invariance, locality, positivity of the energy spectrum and uniqueness of the vacuum can be verified. $\phi$ satisfies the equation of motion,

$$
\begin{aligned}
\frac{1}{i} \gamma^{\mu} \partial_{\mu} \phi(x) & =g \gamma^{\mu}\left[J_{\mu}^{+}(x) \phi(x)+\phi(x) J_{\mu}^{-}(x)\right] \\
& =\lim _{\varepsilon \rightarrow 0} g \gamma^{\mu} \cdot \frac{1}{2}\left[J_{\mu}(x+\varepsilon) \phi(x)+\phi(x) J_{\mu}(x-\varepsilon)\right]
\end{aligned}
$$

where

$$
\begin{aligned}
& J_{\mu}(x)=J_{\mu}^{+}(x)+J_{\mu}^{-}(x)=\frac{1}{\sqrt{\pi}} \partial_{\mu}\left(J^{+}(x)+J^{-}(x)\right), \\
& J^{ \pm}(x)=j^{ \pm}(x)-\alpha \Delta^{ \pm}(x) Q-\beta \tilde{\Delta}^{ \pm}(x) \tilde{Q} .
\end{aligned}
$$

\section{Characterization of Normal Products}

By a normal product of spinor fields $\Psi$ and $\Psi^{\dagger}$ we shall understand an object $N\left[\Psi\left(x_{1}\right) \ldots \Psi\left(x_{m}\right) \Psi^{\dagger}\left(y_{1}\right) \ldots \Psi^{\dagger}\left(y_{n}\right)\right]$ with the following properties.

A. $N\left[\Psi\left(x_{1}\right) \ldots \Psi\left(x_{m}\right) \Psi^{\dagger}\left(y_{1}\right) \ldots \Psi^{\dagger}\left(y_{n}\right)\right]$ is an $(m+n)$-fold operatorvalued tempered distribution which has the same covariant transformation properties with respect to the Poincaré group as

$$
\Psi(x) \ldots \Psi\left(x_{m}\right) \Psi^{\dagger}\left(y_{1}\right) \ldots \Psi^{\dagger}\left(y_{n}\right) .
$$

B. For any test function $f \in \mathscr{S}\left(R^{k}\right), k=$ dimension of space-time,

$$
\int N\left[\Psi\left(x+\varepsilon_{1}\right) \ldots \Psi\left(x+\varepsilon_{m}\right) \Psi^{\dagger}\left(x+\varepsilon_{m+1}\right) \ldots \Psi^{\dagger}\left(x+\varepsilon_{m+n}\right)\right] f(x) d^{k} x
$$

is an operator-valued function of the $\varepsilon_{i}$ which is infinitely differentiable in some neighborhood of the origin $\left(\varepsilon_{i}=0\right.$ for all $\left.i\right)$.

C. The quantities $N\left[\Psi(x) \ldots \Psi(x) \Psi^{\dagger}(x) \ldots \Psi^{\dagger}(x)\right]$

$$
\left.\frac{\partial}{\partial \varepsilon_{i_{1}}^{\mu_{1}}} \cdots \frac{\partial}{\partial \varepsilon_{i_{r}}^{\mu_{r}}} N\left[\Psi\left(x+\varepsilon_{1}\right) \cdots \Psi^{\dagger}\left(x+\varepsilon_{m+n}\right)\right]\right|_{\substack{\varepsilon_{i}=0 \\ i=1,2, \ldots, m+n}}
$$

are local, covariant fields, and are local with respect to $\Psi$ and $\Psi^{\dagger}$.

D. The normal product of any permutation of the factors $\Psi\left(x_{1}\right), \Psi\left(x_{2}\right), \ldots, \Psi^{\dagger}\left(y_{n}\right)$ is equal to $N\left[\Psi\left(x_{1}\right) \ldots \Psi^{\dagger}\left(y_{n}\right)\right]$ multiplied by the sign of the permutation. 
E. The short-distance behavior of the product

$$
\Psi\left(x_{1}\right) \ldots \Psi\left(x_{m}\right) \Psi^{\dagger}\left(y_{1}\right) \ldots \Psi^{\dagger}\left(y_{n}\right)
$$

may be expressed by an expansion in terms of normal products of the basic fields and their derivatives.

The above list of requirements is insufficiently precise to provide a rigorous definition of normal products; rather it is a set of heuristic criteria generalizing some of the well known properties of the Wick products of free fields. In particular, in formulating item (E), we have in mind the reciprocal free-field formulas,

$$
\begin{aligned}
& \psi\left(x_{1}\right) \ldots \psi\left(x_{m}\right) \psi^{\dagger}\left(y_{1}\right) \ldots \psi^{\dagger}\left(y_{n}\right) \\
& =\sum_{S}(-1)^{\delta_{S}}\left\langle 0\left|\psi\left(x_{S 1}\right) \ldots \psi\left(x_{S m_{S}}\right) \psi^{\dagger}\left(y_{S 1}\right) \ldots \psi^{\dagger}\left(y_{S m_{S}}\right)\right| 0\right\rangle \\
& \quad \cdot:\left(x_{T 1}\right) \ldots \psi\left(x_{T m_{T}}\right) \psi^{\dagger}\left(y_{T 1}\right) \ldots \psi^{\dagger}\left(y_{T n_{T}}\right): \\
& \quad: \psi\left(x_{1}\right) \ldots \psi\left(x_{m}\right) \psi^{\dagger}\left(y_{1}\right) \ldots \psi^{\dagger}\left(y_{n}\right): \\
& =\sum_{S}(-1)^{\delta_{S}+m_{S}}\left\langle 0\left|\psi\left(x_{S 1}\right) \ldots \psi\left(x_{S m_{S}}\right) \psi^{\dagger}\left(y_{S 1}\right) \ldots \psi^{\dagger}\left(y_{S m_{S}}\right)\right| 0\right\rangle \\
& \quad \cdot \psi\left(x_{T 1}\right) \ldots \psi\left(x_{T m_{T}}\right) \psi^{\dagger}\left(y_{T 1}\right) \ldots \psi^{\dagger}\left(y_{T n_{T}}\right), \\
& S=\left\{x_{S 1}, \ldots, x_{S m_{S}} ; y_{S 1}, \ldots, y_{S m_{S}}\right\}, \quad S \cup T=X=\left\{x_{1}, \ldots, x_{m} ; y_{1}, \ldots, y_{n}\right\} \\
& T=\left\{x_{T 1}, \ldots, x_{T m_{T}} ; y_{T 1}, \ldots, y_{T n_{T}}\right\}, \quad S \cap T=\emptyset
\end{aligned}
$$

In each case, the sum includes $S=\emptyset$ and $S=X$, and $(-1)^{\delta_{s}}$ is the sign of the relative permutation of the sfinor factors.

Eq. (3.1) gives a complete description of the short-distance behavior of products of free fields. By expanding the

$$
: \psi\left(x_{T 1}\right) \ldots \psi\left(x_{T m_{T}}\right) \psi^{\dagger}\left(y_{T 1}\right) \ldots \psi^{\dagger}\left(y_{T n_{T}}\right):
$$

in Taylor series about $x_{T i}=y_{T j}=X$ (only a finite number of terms are needed, so that no assumption about analyticity, only the infinite differentiability of (B), is used), we arrive at Wilson's expansion (1.3). As a simple example, consider (in two dimensions)

$$
\begin{aligned}
\psi(x) \psi\left(x_{1}\right) \psi^{\dagger}\left(x_{2}\right)= & \psi(x) \psi\left(x+\varepsilon_{1}\right) \psi^{\dagger}\left(x+\varepsilon_{2}\right) \\
= & : \psi(x) \psi\left(x_{1}\right) \psi^{\dagger}\left(x_{2}\right):+\left\langle 0\left|\psi\left(x+\varepsilon_{1}\right) \psi^{\dagger}\left(x+\varepsilon_{2}\right)\right| 0\right\rangle \psi(x) \\
& -\left\langle 0\left|\psi(x) \psi^{\dagger}\left(x+\varepsilon_{2}\right)\right| 0\right\rangle \psi\left(x+\varepsilon_{1}\right) \\
= & : \psi(x) \psi(x) \psi^{\dagger}(x):+\frac{\left(\varepsilon_{1}-\varepsilon_{2}\right)^{\mu} \gamma_{\mu} \gamma^{0}}{2 \pi i\left(\varepsilon_{1}-\varepsilon_{2}\right)^{2}} \psi(x)-\frac{\varepsilon_{2}^{\mu} \gamma_{\mu} \gamma^{0}}{2 \pi i \varepsilon_{2}^{2}} \\
& -\frac{\varepsilon_{2}^{\mu} \varepsilon_{1}^{v} \gamma_{\mu} \gamma^{0}}{2 \pi i \varepsilon_{2}^{2}} \partial_{\nu} \psi(x)+O(\varepsilon) .
\end{aligned}
$$


It is just this sort of analysis which we hope to achieve by defining normal products of Thirring model fields in Sections IV and V.

It is improbable that normal products satisfying all of the criteria (A) $-(E)$ will exist for arbitrary field theories. In particular items (B)-(E) may have to be modified to exclude lightlike $\varepsilon_{i}$ and $\varepsilon_{i}-\varepsilon_{j}$, and item (D) may have to be rejected entirely. We shall see that in the case of the Thirring model it is not necessary to weaken the requirements.

\section{Normal Product of Two Fields}

Before immersing ourselves in the complexities of the most general products of Thirring model fields, it will be instructive to examine in detail the short-distance behavior of the twofold product $\phi\left(x_{1}\right) \phi^{\dagger}\left(x_{2}\right)$. The aim of the present section will be to discover an object $N\left[\phi\left(x_{1}\right) \phi^{\dagger}\left(x_{2}\right)\right]$ which satisfies the conditions (A)-(E) of Section III. Our standards of mathematical rigor will be essentially those adopted by Klaiber in the construction of the model [3]: we shall be working within the framework of a specific Hilbert space, the Fock space of the free spinor field in two dimensions, and we shall give close attention to the distributional character of the fields and their matrix elements.

\section{a) Heuristic Derivation of the Normal Product}

From the definitions of $\phi$ and $\phi^{\dagger}$ and the commutation relations of Section II, Part D, we may write

$\phi_{\alpha_{1}}\left(x_{1}\right) \phi_{\alpha_{2}}^{\dagger}\left(x_{2}\right)$

$=\left(-\mu^{2} \varepsilon_{12}^{2}+i \eta \varepsilon_{12}^{0}\right)^{-\frac{a+b \gamma_{1}^{5} \gamma_{2}^{5}}{4 \pi}} e^{i\left(\chi^{+}\left(x_{1}\right)-\chi^{+}\left(x_{2}\right)\right)} \psi_{\alpha_{1}}\left(x_{1}\right) \psi_{\alpha_{2}}^{\dagger}\left(x_{2}\right) e^{i\left(\chi^{-}\left(x_{1}\right)-\chi^{-}\left(x_{2}\right)\right)}$

where

$$
\begin{aligned}
& \varepsilon_{12}=x_{1}-x_{2}, \quad \mu=e^{-\Gamma^{\prime}(1)} x, \quad \eta \rightarrow 0+, \\
& \begin{array}{l}
a=\alpha^{2}-2 \alpha \sqrt{\pi}, \\
b=\beta^{2}-2 \beta \sqrt{\pi},
\end{array} \quad \gamma_{i}^{5}=\left\{\begin{array}{ll}
-1 & \alpha_{i}=1 \\
+1 & \alpha_{i}=2,
\end{array} \quad i=1,2 .\right.
\end{aligned}
$$

There are two obvious sources of singularities of $\phi\left(x_{1}\right) \phi^{\dagger}\left(x_{2}\right)$ in $\varepsilon_{12}^{2}$ as $\varepsilon_{12}^{2} \rightarrow 0:$ the factor

$$
\left(-\mu^{2} \varepsilon_{12}^{2}+i \eta \varepsilon_{12}^{0}\right)^{-\frac{a+b \gamma_{1}^{5} \gamma_{2}^{5}}{4 \pi}}
$$

and the vacuum expectation value of $\psi\left(x_{1}\right) \psi^{\dagger}\left(x_{2}\right)$ :

$$
\begin{aligned}
\psi\left(x_{1}\right) \psi^{\dagger}\left(x_{2}\right)=: & : \psi\left(x_{1}\right) \psi^{\dagger}\left(x_{2}\right):+\left\langle 0\left|\psi\left(x_{1}\right) \psi^{\dagger}\left(x_{2}\right)\right| 0\right\rangle, \\
\left\langle 0\left|\psi\left(x_{1}\right) \psi^{\dagger}\left(x_{2}\right)\right| 0\right\rangle & =\frac{1}{2 \pi i} \frac{\varepsilon_{12}^{\mu} \gamma_{\mu} \gamma^{0}}{\varepsilon_{12}^{2}-i \eta \varepsilon_{12}^{0}} .
\end{aligned}
$$


By removing these singularities in a suitable manner we hope to construct an object $N\left[\phi\left(x_{1}\right) \phi^{\dagger}\left(x_{2}\right)\right]$ which has the same covariance properties as $\phi\left(x_{1}\right) \phi^{\dagger}\left(x_{2}\right)$, and which approaches a covariant field in the limit $\varepsilon_{12} \rightarrow 0$, with the limiting field independent of the space-time direction of $\varepsilon_{12}$. A first guess might be

$$
\begin{aligned}
N_{a}\left[\phi\left(x_{1}\right) \phi^{\dagger}\left(x_{2}\right)\right]= & \left(-\mu^{2} \varepsilon_{12}^{2}+i \eta \varepsilon_{12}^{0}\right)^{\frac{a+b \gamma_{1}^{5} \gamma_{2}^{5}}{4 \pi}} \phi\left(x_{1}\right) \phi^{\dagger}\left(x_{2}\right) \\
& -\left\langle 0\left|\psi\left(x_{1}\right) \psi^{\dagger}\left(x_{2}\right)\right| 0\right\rangle
\end{aligned}
$$

or, possibly,

$$
\begin{aligned}
N_{b}\left[\phi\left(x_{1}\right) \phi^{\dagger}\left(x_{2}\right)\right]= & \left(-\mu^{2} \varepsilon_{12}^{2}+i \eta \varepsilon_{12}^{0}\right)^{\frac{a+b \gamma_{1}^{5} \gamma_{2}^{5}}{4 \pi}} \phi\left(x_{1}\right) \phi^{\dagger}\left(x_{2}\right) \\
& -\left\langle 0\left|\psi\left(x_{1}\right) \psi^{\dagger}\left(x_{2}\right)\right| 0\right\rangle e^{i\left(\chi^{+}\left(x_{1}\right)-\chi^{+}\left(x_{2}\right)\right)} e^{i\left(\chi^{-}\left(x_{1}\right)-\chi^{-}\left(x_{2}\right)\right)} .
\end{aligned}
$$

Actually, neither $N_{a}$ nor $N_{b}$ has the correct properties, although both prescriptions succeed in removing the singularities (4.2) and (4.3). Expression (4.5) can be eliminated from consideration immediately, since the second term is not covariant: the quantity $\chi^{ \pm}\left(x_{1}\right)-\chi^{ \pm}\left(x_{2}\right)$ does not transform as a scalar, due to the presence of terms proportional to $\Delta^{ \pm}\left(x_{i}\right)$ and $\tilde{\Delta}^{ \pm}\left(x_{i}\right)$. Although $N_{a}\left[\phi\left(x_{1}\right) \phi^{\dagger}\left(x_{1}\right)\right]$ does not suffer from this difficulty, it is easy to see that it does not approach a unique limit for vanishing $\varepsilon_{1}$ and $\varepsilon_{2}$ :

$$
\begin{aligned}
N_{a}\left[\phi\left(x_{1}\right) \phi^{\dagger}\left(x_{2}\right)\right] \rightarrow & e^{i\left(\chi_{1}^{\dagger}(x)-\chi \chi^{\ddagger}(x)\right)}: \psi(x) \psi^{\dagger}(x): e^{i\left(x \bar{\Gamma}(x)-\chi_{\overline{2}}^{-}(x)\right)} \\
& \quad \lim _{\varepsilon_{12} \rightarrow 0} \frac{\varepsilon_{12}^{\mu} \varepsilon_{12}^{v}}{2 \pi i \varepsilon_{12}^{2}} \gamma_{\mu} \gamma^{0} \partial_{\nu} \chi(x) .
\end{aligned}
$$

The second term in (4.6) contains contributions proportional to

$$
\frac{\varepsilon_{12}^{\mu} \varepsilon_{12}^{v}}{\varepsilon_{12}^{2}} \gamma_{\mu} \gamma^{0}\left(\alpha J_{v}+\beta \gamma^{5} \tilde{J}_{v}\right)
$$

which, apart from being singular on the light cone, do not approach direction-independent limits for $\varepsilon_{12} \rightarrow 0\left(\varepsilon_{12}^{2}>0\right.$ or $\left.\varepsilon_{12}^{2}<0\right)$.

It turns out that the correct regularization procedure is the following. Writing

we define

$$
\begin{aligned}
\chi^{ \pm}(x) & =K^{ \pm}(x)+\Omega^{ \pm}(x) \\
K^{ \pm}(x) & =\alpha J^{ \pm}(x)+\beta \gamma^{5} \tilde{J}^{ \pm}(x) \\
\Omega^{ \pm}(x) & =\sqrt{\pi}\left(\alpha Q+\beta \gamma^{5} \tilde{Q}\right)\left(\Delta^{ \pm}(x)+\gamma^{5} \tilde{\Delta}^{ \pm}(x)\right) \\
\Omega(x) & =\Omega^{+}(x)+\Omega^{-}(x)
\end{aligned}
$$

$$
\begin{aligned}
& N\left[\phi\left(x_{1}\right) \phi^{\dagger}\left(x_{2}\right)\right]=\left(-\mu^{2} \varepsilon_{12}^{2}+i \eta \varepsilon_{12}^{0}\right)^{\frac{a+b \gamma_{1}^{5} \gamma_{2}^{5}}{4 \pi}} \\
& {\left[\phi\left(x_{1}\right) \phi^{\dagger}\left(x_{2}\right)-\left\langle 0\left|\phi\left(x_{1}\right) \phi^{\dagger}\left(x_{2}\right)\right| 0\right\rangle e^{i\left(K^{+}\left(x_{1}\right)-K^{+}\left(x_{2}\right)\right)} e^{i\left(K^{-}\left(x_{1}\right)-K^{-}\left(x_{2}\right)\right)}\right] .}
\end{aligned}
$$


Then

$N\left[\phi\left(x_{1}\right) \phi^{\dagger}\left(x_{2}\right)\right]=e^{i\left(\chi^{+}\left(x_{1}\right)-\chi^{+}\left(x_{2}\right)\right)}: \psi\left(x_{1}\right) \psi^{\dagger}\left(x_{2}\right): e^{i\left(\chi^{-}\left(x_{1}\right)-\chi^{-}\left(x_{2}\right)\right)}$

$+\left(e^{i\left(\Omega\left(x_{1}\right)-\Omega\left(x_{2}\right)\right)}-1\right) e^{i\left(K^{+}\left(x_{1}\right)-K^{+}\left(x_{2}\right)\right)} e^{i\left(K^{-}\left(x_{1}\right)-K^{-}\left(x_{2}\right)\right)}\left\langle 0\left|\psi\left(x_{1}\right) \psi^{\dagger}\left(x_{2}\right)\right| 0\right\rangle$.

The singularities (4.2) and (4.3) have clearly been removed, and for vanishing $\varepsilon_{i}$ we obtain the (limit

$$
\begin{aligned}
N\left[\phi \phi^{\dagger}\right](x) & =\lim _{\varepsilon_{i} \rightarrow 0}\left\{e^{i\left(\chi^{+}\left(x_{1}\right)-\chi^{+}\left(x_{2}\right)\right)}: \psi\left(x_{1}\right) \psi^{\dagger}\left(x_{2}\right): e^{i\left(\chi^{-}\left(x_{1}\right)-\chi^{-}\left(x_{2}\right)\right)}\right. \\
+ & \left.\frac{\varepsilon_{12}^{v} \varepsilon_{12}^{\mu}}{2 \pi \varepsilon_{12}^{2}} \gamma_{\mu} \gamma^{0} \partial_{\nu} \Omega(x) e^{i\left(K^{+}\left(x_{1}\right)-K^{+}\left(x_{2}\right)\right)} e^{i\left(K^{-}\left(x_{1}\right)-K^{-}\left(x_{2}\right)\right)}\right\}
\end{aligned}
$$

To simplify this expression we use

to obtain

$$
\begin{aligned}
\gamma^{5} \gamma^{\mu} & =-\varepsilon^{\mu v} \gamma_{\mu}, \\
\partial_{v} \tilde{\Delta}(x) & =\varepsilon_{v \varrho} \partial^{\varrho} \Delta(x), \\
\varepsilon_{v \varrho} \varepsilon^{\mu \sigma} & =\delta_{v}^{\sigma} \delta_{\varrho}^{\mu}-\delta_{v}^{\mu} \delta_{\varrho}^{\sigma}
\end{aligned}
$$

$$
\varepsilon_{12}^{\mu} \varepsilon_{12}^{v} \gamma_{v_{A}}^{\gamma^{0}} \partial_{\mu} \Omega(x)=\varepsilon_{12}^{2} \sqrt{\pi}\left(\alpha Q+\beta \gamma^{5} \tilde{Q}\right) \partial_{\mu} \Delta(x) \gamma^{\mu} \gamma^{0}
$$

and hence

$$
\begin{aligned}
N\left[\phi \phi^{\dagger}\right](x)= & e^{i\left(\chi^{+}(x)-\chi^{+}(x)\right)}: \psi \psi^{\dagger}:(x) e^{i\left(\chi^{\overline{1}}(x)-\chi_{\overline{2}}(x)\right)} \\
& +\frac{\gamma^{\mu} \gamma_{0}}{2 \pi} \partial_{\mu} \Delta(x)\left(\alpha Q+\beta \gamma^{5} \tilde{Q}\right),
\end{aligned}
$$

where $\chi_{1}^{ \pm}(x)$ is independent of $\varepsilon_{i}$ but remains a function of $\gamma_{i}^{5}$. Thus $N\left[\phi\left(x_{1}\right) \phi^{\dagger}\left(x_{2}\right)\right]$ approaches a limiting field which does not depend on the space-time direction of $\varepsilon_{i}$. To see that it is also translation and Lorentz invariant, we need only to verify, for any element $(a, \Lambda)$ of the Poincare group, and for $\gamma_{1}^{5}=\gamma_{2}^{5}$,

$$
\begin{array}{r}
U(a, \Lambda)\left(K^{ \pm}\left(x_{1}\right)-K^{ \pm}\left(x_{2}\right)\right) U(a, \Lambda)^{-1} \\
=K^{ \pm}\left(\Lambda x_{1}+a\right)-K^{ \pm}\left(\Lambda x_{2}+a\right),
\end{array}
$$

and hence, from Eq. (4.7),

$$
\begin{array}{r}
U(a, \Lambda) N\left[\phi\left(x_{1}\right) \phi^{\dagger}\left(x_{2}\right)\right] U(a, \Lambda)^{-1} \\
=e^{-\frac{1}{2}\left(\gamma_{1}^{5}+\gamma_{2}^{5}\right) \chi} N\left[\phi\left(\Lambda x_{1}+a\right) \phi^{\dagger}\left(\Lambda x_{2}+a\right)\right]
\end{array}
$$

where

$$
\Lambda=\left(\begin{array}{cc}
\cosh \chi & \sinh \chi \\
\sinh \chi & \cosh \chi
\end{array}\right)
$$


But Eq. (4.11) is easily derived from

$$
J^{-}|0\rangle=0=\tilde{J}^{-}|0\rangle
$$

$\left[J^{-}(x), \phi(y)\right]$

$$
=\left[(\alpha-\sqrt{\pi})\left(D^{-}(x-y)+\Delta^{+}(y)\right)+(\beta-\sqrt{\pi}) \gamma^{5}\left(\tilde{D}^{-}(x-y)+\tilde{\Delta}^{+}(y)\right)\right] \phi(y),
$$$$
\left[\tilde{J}^{-}(x), \phi(y)\right]
$$$$
=\left[(\alpha-\sqrt{\pi})\left(\tilde{D}^{-}(x-y)+\tilde{\Delta}^{+}(y)\right)+(\beta-\sqrt{\pi}) \gamma^{5}\left(D^{-}(x-y)+\Delta^{+}(y)\right)\right] \phi(y),
$$

$$
D^{-}(\Lambda \xi+a)=D^{-}(\xi), \quad \tilde{D}^{-}(\Lambda \xi+a)=\tilde{D}(\xi)+\frac{i}{2 \pi} \chi .
$$

The local commutation relations for $N\left[\phi \phi^{\dagger}\right]$ follow trivially from those of $\phi, \phi^{\dagger}, J_{\mu}$ and $\tilde{J}_{\mu}$, using (4.7). It is also straightforward to verify

$$
\begin{aligned}
& N\left[\phi^{\dagger}\left(x_{2}\right) \phi\left(x_{1}\right)\right] \equiv\left(-\mu^{2} \varepsilon_{12}^{2}-i \eta \varepsilon_{12}^{0}\right)^{\frac{a+b \gamma_{1}^{5} \gamma_{2}^{5}}{4 \pi}} \phi^{\dagger}\left(x_{2}\right) \phi\left(x_{1}\right) \\
& -\left\langle 0\left|\psi^{\dagger}\left(x_{2}\right) \psi\left(x_{1}\right)\right| 0\right\rangle e^{i\left(K^{+}\left(x_{1}\right)-K^{+}\left(x_{2}\right)\right)} e^{i\left(K^{-}\left(x_{1}\right)-K^{-}\left(x_{2}\right)\right)}=-N\left[\phi\left(x_{1}\right) \phi^{\dagger}\left(x_{2}\right)\right]
\end{aligned}
$$

To complete our heuristic discussion of the suitability of $N\left[\phi\left(x_{1}\right) \phi^{\dagger}\left(x_{2}\right)\right]$ as a normal product, we must show that, at least formally, it is infinitely differentiable in the vicinity of the origin. But this is easily seen from Eq. (4.8). Thus, we define

$$
\begin{aligned}
& N\left[\partial_{\mu} \phi\left(x_{1}\right) \phi^{\dagger}\left(x_{2}\right)\right] \equiv \frac{\partial}{\partial \varepsilon_{1}^{\mu}} N\left[\phi\left(x+\varepsilon_{1}\right) \phi^{\dagger}\left(x+\varepsilon_{2}\right)\right] \\
& =e^{i\left(\chi^{+}\left(x_{1}\right)-\chi^{+}\left(x_{2}\right)\right)}\left[\begin{array}{l}
\partial_{\mu} \psi\left(x_{1}\right) \psi^{\dagger}\left(x_{2}\right):+i \partial_{\mu} \chi^{+}\left(x_{1}\right): \psi\left(x_{1}\right) \psi^{+}\left(x_{2}\right): \\
+\mathrm{i}: \psi\left(x_{1}\right) \psi^{\dagger}\left(x_{2}\right): \partial_{\mu} \chi^{-}\left(x_{1}\right)
\end{array}\right] \\
& \cdot e^{i\left(\chi^{-}\left(x_{1}\right)-\chi^{-}\left(x_{2}\right)\right)} \\
& +e^{i\left(K^{+}\left(x_{1}\right)-K^{+}\left(x_{2}\right)\right)}\left[\begin{array}{l}
i \sqrt{\pi} K_{\mu}\left(x_{1}\right)\left\langle 0\left|\psi\left(x_{1}\right) \psi^{\dagger}\left(x_{2}\right)\right| 0\right\rangle\left(e^{i\left(\Omega\left(x_{1}\right)-\Omega\left(x_{2}\right)\right)}-1\right) \\
+\frac{\partial}{\partial x_{1}^{\mu}}\left(\left\langle 0\left|\psi\left(x_{1}\right) \psi^{\dagger}\left(x_{2}\right)\right| 0\right\rangle\left(e^{i\left(\Omega\left(x_{1}\right)-\Omega\left(x_{2}\right)\right)}-1\right)\right)
\end{array}\right] \\
& \cdot e^{i\left(K^{-}\left(x_{1}\right)-K^{-}\left(x_{2}\right)\right)}
\end{aligned}
$$

which approaches the local, covariant limit (independent of direction), $N\left[\partial_{\mu} \phi \phi^{\dagger}\right](x)$

$$
\begin{aligned}
= & e^{i\left(\chi^{+}(x)-\chi^{+}(x)\right)}\left[\begin{array}{l}
\partial_{\mu} \psi \psi^{\dagger}:(x)+i \partial_{\mu} \chi_{1}^{-}(x): \psi \psi^{\dagger}:(x) \\
+i: \psi \psi^{\dagger}:(x) \partial_{\mu} \chi_{1}^{-}(x)
\end{array}\right] e^{i\left(x_{1}^{-}(x)-\chi_{\overline{2}}^{-}(x)\right)} \\
& +\frac{\gamma^{\lambda} \gamma_{0}}{2 \pi}\left[i \sqrt{\pi} K_{\mu}(x) \partial_{\lambda} \Delta(x)+\frac{1}{2} \partial_{\lambda} \partial_{\mu} \Delta(x)+\frac{i}{2} \partial_{\lambda} \Omega(x) \partial_{\mu} \Delta(x)\right] \\
& \cdot\left[\alpha Q+\beta \gamma^{5} \tilde{Q}\right] .
\end{aligned}
$$


By differentiating further Eq. (4.8), we may define derivatives of arbitrary order:

$$
\begin{aligned}
& N\left[\frac{\partial^{(m)} \phi\left(x_{1}\right)}{\partial x_{1}^{\mu_{1}} \ldots \partial x_{1}^{\mu_{m}}} \frac{\partial^{(n)} \phi\left(x_{2}\right)}{\partial x_{2}^{v_{1}} \ldots \partial x_{2}^{\nu_{n}}}\right] \\
& \equiv \frac{\partial^{(m)}}{\partial \varepsilon_{1}^{\mu_{1}} \ldots \partial \varepsilon_{1}^{\mu_{m}}} \frac{\partial^{(n)}}{\partial \varepsilon_{2}^{\nu_{1}} \ldots \partial \varepsilon_{2}^{v_{n}}} N\left[\phi\left(x+\varepsilon_{1}\right) \phi^{\dagger}\left(x+\varepsilon_{2}\right)\right] .
\end{aligned}
$$

As our notation indicates, the derivatives of $N\left[\phi\left(x_{1}\right) \phi^{\dagger}\left(x_{2}\right)\right]$ provide a natural means of defining a normal-product regularization of

$$
\frac{\partial^{(m)} \phi\left(x_{1}\right)}{\partial x_{1}^{\mu_{1}} \ldots \partial x_{1}^{\mu_{m}}} \frac{\partial^{(n)} \phi^{\dagger}\left(x_{2}\right)}{\partial x_{2}^{v_{1}} \ldots \partial x_{2}^{v_{n}}} \text {. }
$$

\section{b) Mathematical Status of the Normal Product}

We now make more precise some of the rather formal manipulations of Part A by means of the following lemmas:

Lemma 1. The objects

and

$$
N\left[\phi\left(x+\varepsilon_{1}\right) \phi^{\dagger}\left(x+\varepsilon_{2}\right)\right]
$$

$$
N\left[\left(\partial_{\mu_{1}} \ldots \partial_{\mu_{m}} \phi\left(x+\varepsilon_{1}\right)\right)\left(\partial_{v_{1}} \ldots \partial_{v_{n}} \phi^{\dagger}\left(x+\varepsilon_{2}\right)\right)\right],
$$

given formally by Eqs. (4.8) and (4.15) for arbitrary fixed two-vectors $\varepsilon_{1}$ and $\varepsilon_{2}$, define operator-valued tempered distributions in the variable $x$, with the common dense domain $\mathscr{D}_{\phi}$.

Lemma 2. Expressions (4.7) and (4.8) are equivalent definitions of $N\left[\phi\left(x_{1}\right) \phi^{\dagger}\left(x_{2}\right)\right]$.

Lemma 3. For all two-vectors $\varepsilon_{1}$ and $\varepsilon_{2}$, and for all $\Psi \in \mathscr{D}_{\phi}, f \in \mathscr{S}\left(R^{2}\right)$, $\left(\int f(x) N\left[\phi\left(x+\varepsilon_{1}\right) \phi^{\dagger}\left(x+\varepsilon_{2}\right)\right] d^{2} x\right) \Psi$ is infinitely differentiable in $\varepsilon_{i}$ in the strong topology.

Proof of Lemma 1. At the very outset we are confronted with the following difficulty: assuming that $N\left[\phi\left(x_{1}\right) \phi^{\dagger}\left(x_{2}\right)\right]$ is an operatorvalued distribution in $x_{1}$ and $x_{2}$, how do we give precise meaning to the expression $\int f(x) N\left[\phi\left(x+\varepsilon_{1}\right) \phi^{\dagger}\left(x+\varepsilon_{2}\right)\right] d^{2} x$ ? Here we shall follow Klaiber's example and plunge ahead, hoping that the explicit calculation of matrix elements will indicate the sense of the products of distributions involved.

To verify Lemma 1 , we choose an arbitrary state $\Psi \in \mathscr{D}_{\phi}$ (we recall that $\mathscr{D}_{\phi}$ consists of all states formed by applying a polynomial in $\phi$ and $\phi^{\dagger}$ on the vacuum state), as well as arbitrary two-vectors $\varepsilon_{1}$ and $\varepsilon_{2}$ and 
test function $f \in \mathscr{S}\left(R^{0}\right)$. We wish to show that

$$
\begin{aligned}
{\left[\int d^{2} x f(x) e^{i\left(\chi^{+}\left(x+\varepsilon_{1}\right)-\chi^{+}\left(x+\varepsilon_{2}\right)\right)}:\right.} & : \psi\left(x+\varepsilon_{1}\right) \psi^{\dagger}\left(x+\varepsilon_{2}\right): \\
& \left.\cdot e^{i\left(\chi^{-}\left(x+\varepsilon_{1}\right)-\chi^{-}\left(x+\varepsilon_{2}\right)\right)}\right] \Psi
\end{aligned}
$$

and

$$
\begin{gathered}
{\left[\int d^{2} x f(x)\left(e^{i\left(\Omega\left(x+\varepsilon_{1}\right)-\Omega\left(x+\varepsilon_{2}\right)\right)}-1\right)\left\langle 0\left|\psi\left(x+\varepsilon_{1}\right) \psi^{\dagger}\left(x+\varepsilon_{2}\right)\right| 0\right\rangle\right.} \\
\left.\cdot e^{i\left(K^{+}\left(x+\varepsilon_{1}\right)-K^{+}\left(x+\varepsilon_{2}\right)\right)} e^{i\left(K^{-}\left(x+\varepsilon_{1}\right)-K\left(x+\varepsilon_{2}\right)\right)}\right] \Psi
\end{gathered}
$$

have finite norms. We consider typical contributions to the squares of the norms:

$$
\begin{aligned}
& I_{1}\left(\varepsilon_{1}, \varepsilon_{2}, \varepsilon_{1}^{\prime}, \varepsilon_{2}^{\prime}\right)=\int d^{2} x d^{2} y d^{2 m} u d^{2 m} v \overline{f(x)} f(y) \overline{g\left(u_{1}, \ldots, u_{m}\right)} h\left(v_{1}, \ldots, v_{m}\right) \\
& \quad \cdot\langle 0| \phi\left(u_{1}\right) \ldots \phi\left(u_{m}\right) e^{i\left(\chi^{+}\left(x_{2}\right)-\chi^{+}\left(x_{1}\right)\right)}: \psi\left(x_{2}\right) \psi^{\dagger}\left(x_{1}\right): e^{i\left(\chi^{-}\left(x_{2}\right)-\chi^{-}\left(x_{1}\right)\right)} \\
& \quad \cdot e^{i\left(x^{+}\left(y_{1}\right)-\chi^{+}\left(y_{2}\right)\right)}: \psi\left(y_{1}\right) \psi^{\dagger}\left(y_{2}\right): e^{i\left(x^{-}\left(y_{1}\right)-\chi^{-}\left(y_{2}\right)\right)} \phi^{\dagger}\left(v_{1}\right) \ldots \phi^{\dagger}\left(v_{m}\right)|0\rangle \\
& I_{2}\left(\varepsilon_{1}, \varepsilon_{2}, \varepsilon_{1}^{\prime}, \varepsilon_{2}^{\prime}\right)=\int d^{1} x d^{2} y d^{2 m} u d^{2 n} v \overline{f(x)} f(y) \overline{g\left(u_{1}, \ldots, u_{m}\right)} h\left(v_{1}, \ldots v_{m}\right) \\
& \quad \cdot\langle 0| \phi\left(u_{1}\right) \ldots \phi\left(u_{m}\right)\left(e^{i\left(\Omega\left(x_{2}\right)-\Omega\left(x_{1}\right)\right)}-1\right) e^{i\left(K^{+}\left(x_{2}\right)-K^{+}\left(x_{1}\right)\right)} e^{i\left(K^{-}\left(x_{2}\right)-K^{-}\left(x_{1}\right)\right)} \\
& \quad \cdot e^{i\left(K^{+}\left(y_{1}\right)-K^{+}\left(y_{2}\right)\right)} e^{i\left(K^{-}\left(y_{1}\right)-K^{-}\left(y_{2}\right)\right)}\left(e^{i\left(\Omega\left(y_{1}\right)-\Omega\left(y_{2}\right)\right)}-1\right) \phi^{\dagger}\left(v_{1}\right) \ldots \phi^{\dagger}\left(v_{m}\right)|0\rangle
\end{aligned}
$$

where

\section{Defining}

$$
\begin{array}{ll}
x_{1}=x+\varepsilon_{1}, & y_{1}=y+\varepsilon_{1}, \\
x_{2}=x+\varepsilon_{2}, & y_{2}=y+\varepsilon_{2}^{\prime},
\end{array}, \quad g, h \in \mathscr{S}\left(R^{2 m}\right)
$$

$$
\begin{aligned}
d^{ \pm}(x, y)= & \exp \left\{i\left(a+b \gamma_{x}^{5} \gamma_{y}^{5}\right) D^{ \pm}(x-y)\right\}, \\
d_{1}^{ \pm}(x, y)= & \exp \left\{-i\left[\left(\alpha \sqrt{\pi}+\beta \sqrt{\pi} \gamma_{x}^{5} \gamma_{y}^{5}\right) D_{x}^{ \pm}(x, y)\right.\right. \\
& +\left(\alpha \sqrt{\pi} \gamma_{x}^{5}+\beta \sqrt{\pi} \gamma_{y}^{5}\right) \tilde{D}_{x}^{ \pm}(x, y) \\
& +\left(\alpha(\sqrt{\pi}-\alpha)+\beta(\sqrt{\pi}-\beta) \gamma_{x}^{5} \gamma_{y}^{5}\right) \Delta^{ \pm}(x) \\
& \left.\left.-\sqrt{\pi}\left(\alpha \gamma_{x}^{5}+\beta \gamma_{y}^{5}\right) \tilde{\Delta}^{ \pm}(x)\right]\right\} \\
d_{2}^{ \pm}(x, y)= & \exp \left\{i\left[\left(\alpha^{2}+\beta^{2} \gamma_{x}^{5} \gamma_{y}^{5}\right) D_{x x}^{-}(x, y)+\alpha \beta\left(\gamma_{x}^{5}+\gamma_{y}^{5}\right) \tilde{D}_{x x}^{-}(x, y)\right]\right\}, \\
d_{3}(x, y)= & \exp \left\{-i \sqrt{\pi}\left(\alpha+\beta \gamma_{x}^{5} \gamma_{y}^{5}\right)\left(\Delta(x)+\gamma_{x}^{5} \tilde{\Delta}(x)\right)\right\} \\
d_{4}^{ \pm}(x, y)= & \exp \left\{i \left[\left(\alpha(\alpha-\sqrt{\pi})+\beta(\beta-\sqrt{\pi}) \gamma_{x}^{5} \gamma_{y}^{5}\right) D_{x}^{ \pm}(x, y)\right.\right. \\
& \left.\left.+\sqrt{\pi}\left(\alpha \gamma_{x}^{5}+\beta \gamma_{y}^{5}\right) \tilde{D}_{x}^{ \pm}(x, y)\right]\right\}
\end{aligned}
$$

we have

$$
\begin{aligned}
e^{i \chi^{ \pm}(x)} \psi(y) & =d_{1}^{ \pm}(x, y) \psi(y) e^{i \chi^{ \pm}(x)}, \\
e^{i \chi^{-}(x)} e^{i \chi^{+}(y)} & =d_{2}^{-}(x, y) e^{i \chi^{+}(y)} e^{i \chi^{-}(x)} \\
e^{i \Omega(x)} \phi(y) & =d_{3}(x, y) \phi(y) e^{i \Omega(x)} \\
e^{i K^{ \pm}(x)} \phi(y) & =d_{4}^{ \pm}(x, y) \phi(y) e^{i K^{ \pm}(x)}
\end{aligned}
$$


and

$$
d^{-}(x, y)=\frac{d_{2}^{-}(x, y) d_{1}^{-}(x, y)}{d_{1}^{+}(y, x)}
$$

so that

$$
\begin{aligned}
& I_{1}\left(\varepsilon_{1}, \varepsilon_{2} ; \varepsilon_{1}^{\prime}, \varepsilon_{2}^{\prime}\right) \\
& =\int d^{2} x d^{2} y \overline{f(x)} f(y) \int d^{2 m} u d^{2 m} v \overline{g\left(u_{1}, \ldots, u_{m}\right)} h\left(v_{1}, \ldots, v_{m}\right) \\
& . \frac{\prod_{j<i} d^{-}\left(u_{i}-u_{j}\right) d^{-}\left(v_{i}-v_{j}\right)}{\prod_{k, l} d^{-}\left(u_{k}-v_{l}\right)}\left\{\langle 0| \psi\left(u_{1}\right) \ldots \psi\left(u_{m}\right): \psi\left(x_{2}\right) \psi^{\dagger}\left(x_{1}\right):\right. \\
& \cdot: \psi\left(y_{1}\right) \psi^{\dagger}\left(y_{2}\right): \psi^{\dagger}\left(v_{1}\right) \ldots \psi^{\dagger}\left(v_{m}\right)|0\rangle \frac{F\left(u, v, x_{1}\right)}{F\left(u, v, x_{2}\right)} \cdot \frac{G\left(u, v, x, y_{2}\right)}{G\left(u, v, x, y_{1}\right)} \\
& +\left\langle 0\left|\psi\left(u_{1}\right) \ldots \psi\left(u_{m}\right): \psi\left(y_{1}\right) \psi^{\dagger}\left(y_{2}\right): \psi^{\dagger}\left(v_{1}\right) \ldots \psi^{\dagger}\left(v_{m}\right)\right| 0\right\rangle \\
& \cdot\left\langle 0\left|\psi\left(x_{2}\right) \psi^{\dagger}\left(x_{1}\right)\right| 0\right\rangle \frac{F\left(u, v, y_{2}\right)}{F\left(u, v, y_{1}\right)} \frac{J\left(u, v, y, x_{1}\right)}{J\left(u, v, y, x_{2}\right)}\left(\frac{K\left(u, v, y, x_{2}\right)}{K\left(u, v, y, x_{1}\right)}-1\right) \\
& +\left\langle 0\left|\psi\left(u_{1}\right) \ldots \psi\left(u_{m}\right): \psi\left(x_{2}\right) \psi^{\dagger}\left(x_{1}\right): \psi^{\dagger}\left(x_{1}\right) \ldots \psi^{\dagger}\left(v_{m}\right)\right| 0\right\rangle \\
& \cdot\left\langle 0\left|\psi\left(y_{1}\right) \psi^{\dagger}\left(y_{2}\right)\right| 0\right\rangle \frac{F\left(u, v, x_{1}\right)}{F\left(u, v, x_{2}\right)} \cdot \frac{L\left(u, v, x, y_{2}\right)}{L\left(u, v, x, y_{1}\right)} \cdot\left(\frac{K\left(u, v, x, y_{1}\right)}{K\left(u, v, x, y_{2}\right)}-1\right) \\
& +\left\langle 0\left|\psi\left(u_{1}\right) \ldots \psi\left(u_{m}\right) \psi^{\dagger}\left(v_{1}\right) \ldots \psi^{\dagger}\left(v_{m}\right)\right| 0\right\rangle\left\langle 0\left|\psi\left(x_{2}\right) \psi^{\dagger}\left(x_{1}\right)\right| 0\right\rangle \\
& \cdot\left\langle 0\left|\psi\left(y_{1}\right) \psi^{\dagger}\left(y_{2}\right)\right| 0\right\rangle \frac{M\left(u, v, x_{1}\right) M\left(u, v, y_{2}\right)}{M\left(u, v, x_{2}\right) M\left(u, v, y_{1}\right)} \cdot \frac{N\left(x, y_{1}\right)}{N\left(x, y_{2}\right)} \\
& \left.\cdot\left(\frac{K\left(u, v, x, y_{1}\right)}{K\left(u, v, y, x_{1}\right)}-1\right)\left(\frac{K\left(u, v, x, y_{1}\right)}{K\left(u, v, x, y_{2}\right)}-1\right)\right\} \text {, }
\end{aligned}
$$

$$
\begin{aligned}
& I_{2}\left(\varepsilon_{1}, \varepsilon_{2} ; \varepsilon_{1}^{\prime}, \varepsilon_{2}^{\prime}\right) \\
& =\int d^{2} x d^{2} y d^{2 m} u d^{2 m} v \overline{f(x)} f(y) \overline{g\left(u_{1}, \ldots, u_{m}\right)} h\left(v_{1}, \ldots, v_{m}\right) \\
& \quad \cdot\left\langle 0\left|\phi\left(u_{1}\right) \ldots \phi\left(u_{m}\right) \phi^{\dagger}\left(v_{1}\right) \ldots \phi^{\dagger}\left(v_{m}\right)\right| 0\right\rangle\left\langle 0\left|\psi\left(x_{2}\right) \psi^{\dagger}\left(x_{1}\right)\right| 0\right\rangle \\
& \quad \cdot\left\langle 0\left|\psi\left(y_{1}\right) \psi^{\dagger}\left(y_{2}\right)\right| 0\right\rangle \frac{d_{2}^{-}\left(x_{2}, y_{1}\right) d_{2}^{-}\left(x_{1}, y_{2}\right)}{d_{2}^{-}\left(x_{2}, y_{2}\right) d_{2}^{-}\left(x_{1}, y_{1}\right)} \prod_{i} \frac{d_{4}^{-}\left(x_{1}, v_{i}\right) d_{4}^{-}\left(y_{2}, v_{i}\right)}{d_{4}^{-}\left(x_{2}, v_{i}\right) d_{4}^{-}\left(y_{1}, v_{i}\right)}(4.19) \\
& \quad \cdot \frac{d_{4}^{+}\left(x_{1}, u_{i}\right) d_{4}^{+}\left(y_{2}, u_{i}\right)}{d_{4}^{+}\left(x_{2}, u_{i}\right) d_{4}^{+}\left(y_{1}, u_{i}\right)} \cdot\left(\prod_{i} \frac{d_{3}\left(x_{1}, u_{i}\right)}{d_{3}\left(x_{2}, u_{i}\right)}-1\right)\left(\prod_{i} \frac{d_{3}\left(y_{2}, v_{i}\right)}{d_{3}\left(y_{1}, v_{i}\right)}-1\right), \\
& \quad F\left(u, v, x_{k}\right)=\prod_{i} \frac{d^{-}\left(x_{k}-v_{i}\right)}{d^{-}\left(u_{i}-x_{k}\right)}, \quad G\left(u, v, x, y_{k}\right)=F\left(u, v, y_{k}\right) \frac{d^{-}\left(x_{1}-y_{k}\right)}{d^{-}\left(x_{2}-y_{k}\right)}, \\
& \quad J\left(u, v, y, x_{k}\right)=\frac{d_{2}^{-}\left(x_{k}, y_{2}\right)}{d_{2}^{-}\left(x_{k}, y_{1}\right)} \frac{d_{1}^{-}\left(x_{k}, y_{2}\right)}{d_{1}^{-}\left(x_{k}, y_{1}\right)} \prod_{i} \frac{d_{2}^{-}\left(x_{k}, v_{i}\right)}{d_{2}^{-}\left(u_{i}, x_{k}\right)} d_{1}^{+}\left(x_{k}, u_{i}\right) d_{1}^{-}\left(x_{k}, v_{i}\right),
\end{aligned}
$$




$$
\begin{aligned}
K\left(u, v, y, x_{k}\right) & =\prod_{i} \frac{d_{1}^{+}\left(y_{2}, x_{k}\right)}{d_{1}^{+}\left(y_{1}, x_{k}\right)} d_{1}^{-}\left(u_{i}, x_{k}\right) d_{1}^{+}\left(v_{i}, x_{k}\right), \\
L\left(u, v, x, y_{k}\right) & =\frac{d_{2}^{-}\left(x_{1}, y_{k}\right)}{d_{2}^{-}\left(x_{2}, y_{k}\right)} \frac{d_{1}^{+}\left(y_{k}, x_{2}\right)}{d_{1}^{+}\left(y_{k}, x_{1}\right)} \prod_{i} \frac{d_{2}^{-}\left(y_{k}, v_{i}\right)}{d_{2}^{-}\left(u_{i}, y_{k}\right)} d_{1}^{+}\left(y_{k}, u_{i}\right) d_{1}^{-}\left(y_{k}, v_{i}\right), \\
M\left(u, v, x_{k}\right) & =\prod_{i} \frac{d_{2}^{-}\left(x_{k}-v_{i}\right)}{d_{2}^{-}\left(u_{i}-x_{k}\right)} d_{1}^{+}\left(x_{k}, u_{i}\right) d_{1}^{-}\left(x_{k}, v_{i}\right), \\
N\left(x, y_{k}\right) & =\prod_{i} \frac{d_{2}^{-}\left(x_{2}, y_{k}\right)}{d_{2}^{-}\left(x_{1}, y_{k}\right)} .
\end{aligned}
$$

The reader will be able to verify that our placing all $\phi\left(u_{i}\right)$ to the left of all $\phi^{\dagger}\left(v_{j}\right)$ entails no essential loss of generality. We must verify that the quantities

$$
\begin{aligned}
& \frac{\partial^{r}}{\partial \varepsilon_{1}^{\mu_{1}} \ldots \partial \varepsilon_{1}^{\mu_{r}}} \frac{\partial^{5}}{\partial \varepsilon_{2}^{\nu_{1}} \ldots \partial \varepsilon_{2}^{\nu_{5}}} \frac{\partial^{r}}{\partial \varepsilon_{1}^{\mu_{1}} \ldots \partial \varepsilon_{1}^{\mu_{r}}} \\
&\left.\cdot \frac{\partial^{5}}{\partial \varepsilon_{2}^{\prime \nu_{1}} \ldots \partial \varepsilon_{2}^{\prime \nu_{5}}} I_{i}\left(\varepsilon_{1}, \varepsilon_{2}, \varepsilon_{1}^{\prime}, \varepsilon_{2}^{\prime}\right)\right|_{\substack{\varepsilon_{1}=\varepsilon_{1}^{\prime} \\
\varepsilon_{2}=y_{2}}}
\end{aligned}
$$

are finite. To illustrate the procedure, we consider $I_{1}\left(\varepsilon_{1}, \varepsilon_{2} ; \varepsilon_{1}, \varepsilon_{2}\right)$. The first term of expression (4.18) is obviously nonsingular. We write the second term as

$$
E\left(\varepsilon_{1}, \varepsilon_{12}\right)\left\langle 0\left|\psi\left(-\varepsilon_{12}\right) \psi^{\dagger}(0)\right| 0\right\rangle
$$

where $E\left(\varepsilon_{1}, \varepsilon_{12}\right)$ is an infinitely differentiable function of its arguments which vanishes for $\gamma_{x_{1}}^{5}=\gamma_{x_{2}}^{5}=-1, \varepsilon_{12}^{0}+\varepsilon_{12}^{1}=0$ and for $\gamma_{x_{1}}^{5}=\gamma_{x_{2}}^{5}=1$, $\varepsilon_{12}^{0}-\varepsilon_{12}^{1}=0$. Since

$$
\left\langle 0\left|\psi\left(-\varepsilon_{12}\right) \psi^{\dagger}(0)\right| 0\right\rangle=-\left(\begin{array}{cc}
\frac{1}{\varepsilon_{12}^{0}+\varepsilon_{12}^{1}+i \eta} & 0 \\
0 & \frac{1}{\varepsilon_{12}^{0}-\varepsilon_{12}^{1}+i \eta}
\end{array}\right)
$$

we use the mean value theorem on $E\left(\varepsilon_{1}, \varepsilon_{12}\right)$ with respect to $\varepsilon_{12}^{0}+\varepsilon_{12}^{1}$ for $\gamma^{5}=-1$, with respect to $\varepsilon_{12}^{0}-\varepsilon_{12}^{1}$ for $\left.\gamma^{5}=1\right)$ to show that (4.21) is nonsingular on the light cone $\left(\varepsilon_{12}^{0}=-\varepsilon_{12}^{1}\right.$ or $\left.\varepsilon_{12}^{0}=\varepsilon_{12}^{1}\right)$. The third and fourth terms of $I_{1}\left(\varepsilon_{1}, \varepsilon_{2} ; \varepsilon_{1}, \varepsilon_{2}\right)$, as well as other expressions of the form (4.20), may be handled similarly.

Proof (sketch) of Lemma 2. Lemma 2 is verified by direct calculation of $\left\langle\Phi\left|N\left[\phi\left(x_{1}\right) \phi^{\dagger}\left(x_{2}\right)\right]\right| \Psi\right\rangle$ for $\Phi, \Psi \in \mathscr{D}_{\phi}$.

Proof (sketch) of Lemma 3. It is clear from Lemma 1 that

$$
\begin{aligned}
O_{n+1}=\left\{N\left[\phi\left(x_{1}+\delta_{1}\right) \phi^{\dagger}\left(x_{2}+\delta_{2}\right)\right]\right. \text {-Taylor series } \\
\text { to order } \left.n \text { about the points } x_{1}, x_{2}\right\}
\end{aligned}
$$


is a well defined operator-valued tempered distribution. The fact that $\left\|O_{n+1} \Psi\right\|$, for arbitrary $\Psi \in \mathscr{D}_{\phi}$, is of order $n+1$ may be verified by direct calculation.

\section{c) Definition of the Current and Pseudocurrent}

One of the principal motivations for the study of normal products is that it permits the definition of various physical quantities as regularized products of basic fields. In the case of the Thirring model, we may define the current and pseudocurrent by

$$
\begin{aligned}
& J_{\mu}(x)=N\left[\phi^{\dagger} \gamma^{0} \gamma_{\mu} \phi\right](x), \\
& \tilde{J}_{\mu}(x)=N\left[\phi^{\dagger} \gamma^{0} \gamma^{5} \gamma_{\mu} \phi\right](x) .
\end{aligned}
$$

It is easily verified that $J_{\mu}$ and $\tilde{J}_{\mu}$ defined in this way are identical with the current and pseudocurrent defined by (2.3) and

$$
\tilde{J}_{\mu}(x)=\varepsilon_{\mu \nu} J^{v}(x) .
$$

\section{d) Partial Verification of Wilson's Hypothesis}

It is clear from Eq. (4.7) that an expansion (1.3) of $\phi\left(x_{1}\right) \phi^{\dagger}\left(x_{2}\right)$ satisfying all of Wilson's requirements is not possible, thanks to the presence of the dimensionless factor

$$
\left(-\mu^{2} \varepsilon_{12}^{2}+i \eta \varepsilon_{12}^{0}\right)^{-\frac{a+b \gamma_{1}^{5} \gamma_{2}^{5}}{4 \pi}},
$$

which can be made arbitrarily singular by choosing the coupling constant sufficiently large. The object which has a Wilson-type expansion in the Thirring model is not $\phi\left(x_{1}\right) \phi^{\dagger}\left(x_{2}\right)$, but rather

$$
\left(-\mu^{2} \varepsilon_{12}^{2}+i \eta \varepsilon_{12}^{0}\right)^{\frac{a+b \gamma_{1}^{5} \gamma_{2}^{5}}{4 \pi}} \phi\left(x_{1}\right) \phi^{\dagger}\left(x_{2}\right) .
$$

Thus, from (4.7) and (4.22),

$$
\begin{aligned}
( & \left.-\mu^{2} \varepsilon_{12}^{2}+i \eta \varepsilon_{12}^{0}\right)^{\frac{a+b \gamma_{1}^{5} \gamma_{2}^{5}}{4 \pi}} \phi_{\alpha}\left(x_{1}\right) \phi_{\beta}^{\dagger}\left(x_{2}\right) \\
= & N\left[\phi_{\alpha} \phi_{\beta}^{\dagger}\right]\left(x_{1}\right)+\frac{\varepsilon_{12}^{\mu}\left(\gamma_{\mu} \gamma^{0}\right)_{\alpha \beta}}{2 \pi i \varepsilon_{12}^{2}}\left(1+i \sqrt{\pi} \varepsilon_{12}^{v} K_{v}\left(x_{1}\right)\right)+O(\varepsilon) \\
= & \frac{\varepsilon_{12}^{\mu}\left(\gamma_{\mu} \gamma^{0}\right)_{\alpha \beta}}{2 \pi i \varepsilon_{12}^{2}}+\left[\delta_{\alpha \alpha^{\prime}} \delta_{\beta \beta^{\prime}}-\left(\frac{\alpha}{2 \sqrt{\pi}} \frac{\varepsilon_{12}^{\mu} \varepsilon_{12}^{v}}{\varepsilon_{12}^{2}}\right.\right. \\
& \left.\left.+\frac{\beta}{2 \sqrt{\pi}} \frac{\tilde{\varepsilon}_{12}^{\mu} \tilde{\varepsilon}_{12}^{v}}{\tilde{\varepsilon}_{12}^{2}}\right)\left(\gamma_{\mu} \gamma^{0}\right)_{\alpha \beta}\left(\gamma^{0} \gamma_{v}\right)_{\alpha^{\prime} \beta^{\prime}}\right] N\left[\phi_{\alpha^{\prime}} \phi_{\beta^{\prime}}^{\dagger}\right]\left(x_{1}\right)+O(\varepsilon) .
\end{aligned}
$$

19 Commun. math. Phys., Vol. 16 
This equation is to be understood in the following sense: $\varepsilon_{12}=\varepsilon \xi$ for some fixed two-vector $\xi$ not on the light cone, so that $O(\varepsilon)$ is of order $\varepsilon$ for small $\varepsilon$, and $\varepsilon_{12}^{\mu} / \varepsilon_{12}^{2}$ has a $1 / \varepsilon$ singularity.

To obtain a short-distance expansion of $\phi\left(x_{1}\right) \phi^{\dagger}\left(x_{2}\right)$ itself, one can expand

$$
N\left[\phi\left(x_{1}\right) \phi^{\dagger}\left(x_{2}\right)\right] \text { and } e^{i\left(K^{+}\left(x_{1}\right)-K^{+}\left(x_{2}\right)\right)} e^{i\left(K^{-}\left(x_{1}\right)-K^{-}\left(x_{2}\right)\right)}
$$

in Eq. (4.7) in Taylor series. It will be noted, however, that this expansion, for $g$ sufficiently large, will involve many more terms than Wilson's hypothesis would allow. Moreover, it is not immediately apparent how one can reformulate the expressions involving $\mathrm{K}^{+}$and $\mathrm{K}^{-}$in terms of the basic fields. We shall return to this question in Section V.

It is interesting to note that in perturbation theory, to any order in the coupling constant, the Wilson hypothesis is indeed satisfied, since the factor (4.23) would be of the form

$$
\begin{gathered}
1-\lambda \ln \left(-\mu^{2} \varepsilon_{12}^{2}\right)+\frac{\lambda^{2}}{2} \ln ^{2}\left(-\mu^{2} \varepsilon_{12}^{2}\right)+\cdots+\frac{(-\lambda)^{N}}{N !} \ln ^{N}\left(-\mu^{2} \varepsilon_{12}^{2}\right) \\
\lambda=\frac{1}{4 \pi}\left(a+b \gamma_{1}^{5} \gamma_{2}^{5}\right),
\end{gathered}
$$

which is equivalent to unity in Wilson's scheme. The appearance of increasing powers of logarithms as one goes to higher orders is, as Wilson is careful to point out, characteristic also of the renormalized Gell MannLow expansion of Lagrangian perturbation theory. Since in that case one does not attribute a meaning (except formally) to the sum of the infinite perturbation series, it is natural to neglect the presence of the logarithms. However, the above results for the Thirring model (always bearing in mind the special features of the model) indicate that the neglect of logarithms (on which Wilson's dimensional arguments rest) is perhaps one of the finite-order perturbative results which one should not try to carry over to general quantum field theory.

We conclude this section with a simple application of equation (4.24). Referring to (4.22), we may now express the current $J_{\mu}$ as the following limits:

$$
\begin{aligned}
J_{\mu}(x) & =\lim _{\varepsilon_{12} \rightarrow 0} c_{\mu}^{v}\left(\phi^{\dagger}\left(x_{1}\right) \gamma^{0} \gamma_{\nu} \phi\left(x_{2}\right)\left(-\mu^{2} \varepsilon_{12}^{2}+i \eta \varepsilon_{12}^{0}\right)-\frac{\varepsilon_{12 v}}{\pi i \varepsilon_{12}^{2}}\right), \\
c_{\mu}^{v} & =\delta_{\mu}^{v}-\frac{\alpha}{\alpha-\sqrt{\pi}} \frac{\varepsilon_{12 \mu} \varepsilon_{12}^{v}}{\varepsilon_{12}^{2}}-\frac{\beta}{\beta-\sqrt{\pi}} \frac{\tilde{\varepsilon}_{12 \mu} \tilde{\varepsilon}_{12}^{v}}{\tilde{\varepsilon}_{12}^{2}} \text { where } \tilde{\varepsilon}_{\mu}=\varepsilon^{v} \varepsilon_{v \mu} .
\end{aligned}
$$

For the particular choice of an equal-time limit, $c_{\mu}^{v}$ is diagonal with

$$
c_{0}^{0}=\frac{1}{1-\frac{\beta}{\sqrt{\pi}}}, \quad c_{1}^{1}=\frac{1}{1-\frac{\alpha}{\sqrt{\pi}}} .
$$




\section{Normal Product of an Arbitrary Number of Fields}

\section{a) Definition of the Normal Product}

In this section the subtraction procedure of Section IV, Part A, (we shall assume the validity of the semi-formal approach of that subsection) will be generalized in order to define the normal product of an arbitrary number of $\phi$ 's and $\phi^{\dagger}$ 's. In addition we shall obtain a formula for expanding ordinary products in terms of objects slightly more general than the normal products. This expansion is the analogue in the Thirring model of the Wick-product expansion of free-field products, and may be used to carry out a modified form of Wilson's short-distance analysis. As an application we shall show that the equation of motion may be reformulated as a linear relation among normal products.

We consider the product of $m \phi$ 's and $n \phi^{\dagger}$ 's:

$$
\begin{aligned}
& \phi\left(x_{1}\right) \ldots \phi\left(x_{m}\right) \phi^{\dagger}\left(y_{1}\right) \ldots \phi^{\dagger}\left(y_{n}\right) \\
& =f(X) e^{i\left(\sum_{i}^{m} x^{+}\left(x_{i}\right)-\sum_{j}^{n} x^{+}\left(y_{j}\right)\right)} \psi\left(x_{1}\right) \ldots \psi\left(x_{m}\right) \psi^{\dagger}\left(y_{1}\right) \ldots \psi^{\dagger}\left(y_{n}\right) e^{i\left(\sum_{i}^{m} x^{-}\left(x_{i}\right)-\sum_{j}^{n} x^{-}\left(y_{j}\right)\right)}
\end{aligned}
$$

where

$$
\begin{aligned}
f(X)= & \prod_{i<j}^{m}\left[-\mu^{2}\left(x_{i}-x_{j}\right)^{2}+i \eta\left(x_{i}^{0}-x_{j}^{0}\right)\right]^{\frac{a+b \gamma_{i}^{5} \gamma_{j}^{5}}{4 \pi}} \\
& \cdot \prod_{k<l}^{n}\left[-\mu^{2}\left(y_{k}-y_{l}\right)^{2}+i \eta\left(y_{k}^{0}-y_{l}^{0}\right)\right]^{\frac{a+b \gamma_{k}^{5} \gamma_{l}^{5}}{4 \pi}} \\
& \cdot \prod_{r, s}\left[\frac{1}{-\mu^{2}\left(x_{r}-y_{s}\right)^{2}+i \eta\left(x_{r}^{0}-y_{s}^{0}\right)}\right]^{\frac{a+b \gamma_{\gamma}^{5} \gamma_{s}^{5}}{4 \pi}} .
\end{aligned}
$$

Here and in the following we shall restrict ourselves to products with all the $\phi^{\dagger}(y)$ to the right of all the $\phi(x)$. To evaluate products with the $\phi$ 's and $\phi^{\dagger}$ 's in some other order, one has only to permute the factors accordingly in the product of free fields, and make the appropriate changes in $f(X)-\phi^{\dagger}(y)$ standing to the right of $\phi(x)$ contributes a factor $\exp \left\{i\left(a+b \gamma_{x}^{5} \gamma_{y}^{5}\right) D^{-}(x-y)\right\}, \phi(x)$ standing to the right of $\phi^{\dagger}(y)$ contributes $\exp \left\{i\left(a+b \gamma_{x}^{5} \gamma_{y}^{5}\right) D^{-}(y-x)\right\}$ to $f(X)$.

It is convenient to rewrite Eq. (5.1), dividing out the factors

$$
\exp i\left[\sum_{i}^{m} K^{ \pm}\left(x_{i}\right)-\sum_{j}^{n} K^{ \pm}\left(y_{j}\right)\right]
$$


and making a Wick-product expansion of the free-field product:

$$
\begin{aligned}
& \left.e^{-i\left(\sum_{i}^{m} K^{+}\left(x_{i}\right)-\sum_{j}^{n} K^{+}\left(y_{j}\right)\right.}\right) \phi\left(x_{1}\right) \ldots \phi\left(x_{m}\right) \phi^{\dagger}\left(y_{1}\right) \ldots \phi^{\dagger}\left(y_{n}\right) e^{-i\left(\sum_{i}^{m} K^{-}\left(x_{i}\right)-\sum_{j}^{n} K^{-}\left(y_{j}\right)\right)} \\
& \left.=f(X) e^{i\left(\sum_{i}^{m} \Omega^{+}\left(x_{i}\right)-\sum_{j}^{n} \Omega^{+}\left(y_{i}\right)\right.}\right) \psi\left(x_{1}\right) \ldots \psi\left(x_{m}\right) \psi^{\dagger}\left(y_{1}\right) \ldots \psi^{\dagger}\left(y_{n}\right) e^{i\left(\sum_{i}^{m} \Omega^{-}\left(x_{i}\right)-\sum_{j}^{n} \Omega^{-}\left(y_{j}\right)\right)} \\
& \left.=f(X) e^{i\left(\sum_{i}^{m} \Omega\left(x_{i}\right)-\sum_{j}^{n} \Omega\left(y_{j}\right)\right.}\right) \sum_{S}(-1)^{\delta_{S}}\langle 0| \psi\left(x_{S 1}\right) \ldots \psi\left(x_{S m_{S}}\right) \\
& \text { - } \psi^{\dagger}\left(y_{S 1}\right) \ldots \psi^{\dagger}\left(y_{S_{m_{S}}}\right)|0\rangle g(T): \psi\left(x_{T 1}\right) \ldots \psi\left(x_{T m_{T}}\right) \psi^{\dagger}\left(y_{T 1}\right) \ldots \psi^{\dagger}\left(y_{T n_{T}}\right) \text { : }
\end{aligned}
$$

where $S$ and $T$ have the same meaning as in (3.2), and

$$
\begin{aligned}
& g(X)=\exp i\left\{\sum_{i}^{m}\left(\varrho(X)+\sigma(X) \gamma_{x_{i}}^{5}\right)-\sum_{j}^{n}\left(\varrho(X)+\sigma(X) \gamma_{y_{j}}^{5}\right)\right\}, \\
& \varrho(X)=\alpha \sqrt{\pi}\left\{\sum_{i}^{m}\left(\Delta^{-}\left(x_{i}\right)+\gamma_{x_{i}}^{5} \tilde{\Delta}^{-}\left(x_{i}\right)\right)-\sum_{j}^{n}\left(\Delta^{-}\left(y_{j}\right)+\gamma_{\gamma_{j}}^{5} \tilde{\Delta}^{-}\left(y_{j}\right)\right)\right\}, \\
& \sigma(X)=\beta \sqrt{\pi}\left\{\sum_{i}^{m}\left(\tilde{\Delta}^{-}\left(x_{i}\right)+\gamma_{x_{i}}^{5} \Delta^{-}\left(x_{i}\right)\right)-\sum_{j}^{n}\left(\tilde{\Delta}^{-}\left(y_{i}\right)+\gamma_{y_{j}}^{5} \Delta^{-}\left(y_{j}\right)\right)\right\} .
\end{aligned}
$$

The commutation relations

$$
\begin{aligned}
& \psi(x) e^{i a Q}=e^{i a(Q+1)} \psi(x), \\
& \psi(x) e^{i a \tilde{Q}}=e^{i a\left(\tilde{Q}+\gamma^{5}\right)} \psi(x)
\end{aligned}
$$

have been used to arrive at the last line of (5.2).

We are now in a position to define the normal product

$$
N\left[\phi\left(x_{1}\right) \ldots \phi\left(x_{m}\right) \phi^{\dagger}\left(y_{1}\right) \ldots \phi^{\dagger}\left(y_{n}\right)\right],
$$

proceeding in analogy with the definition (4.7) of $N\left[\phi(x) \phi^{\dagger}(y)\right]$ :

$$
\begin{aligned}
N[ & {\left[\phi\left(x_{1}\right) \ldots \phi\left(x_{m}\right) \phi^{\dagger}\left(y_{1}\right) \ldots \phi^{\dagger}\left(y_{n}\right)\right] } \\
= & \sum_{S}(-1)^{\delta_{S}+m_{S}}\left\langle 0\left|\phi\left(x_{S 1}\right) \ldots \phi\left(x_{S m_{S}}\right) \phi^{\dagger}\left(y_{S 1}\right) \ldots \phi^{\dagger}\left(y_{S m_{S}}\right)\right| 0\right\rangle f(s)^{-1} \\
& \cdot f(T)^{-1} e^{i\left(\sum_{i}^{\left.m_{S} K^{+}\left(x_{S i}\right)-\sum_{j}^{m_{S}} K^{+}\left(y_{S j}\right)\right)} \phi\left(x_{T 1}\right) \ldots \phi\left(x_{T m_{T}}\right)\right.} \\
& \cdot \phi^{\dagger}\left(y_{T 1}\right) \ldots \phi^{\dagger}\left(y_{T n_{T}}\right) e^{i\left(\sum_{i}^{m_{S} K^{-}\left(x_{S i}\right)-\sum_{j}^{m_{S}} K^{-}\left(y_{S j}\right)}\right)}
\end{aligned}
$$

where $S, T$ and $(-1)^{\delta_{s}}$ have the same meaning as in (3.2).

To show that $N\left[\phi\left(x_{1}\right) \ldots \phi\left(x_{m}\right) \phi^{\dagger}\left(y_{1}\right) \ldots \phi^{\dagger}\left(y_{n}\right)\right]$ is nonsingular in $\varepsilon_{i}=x_{i}-x, \varepsilon_{m+j}=y_{j}-x$ as $\varepsilon_{k} \rightarrow 0$, and in fact approaches a limit in- 
dependent of the choice of the $\varepsilon_{k}$, we use Eq. (5.2) to write

$$
\begin{aligned}
& N\left[\phi\left(x_{1}\right) \ldots \phi\left(x_{m}\right) \phi^{\dagger}\left(y_{1}\right) \ldots \phi^{\dagger}\left(y_{n}\right)\right]
\end{aligned}
$$

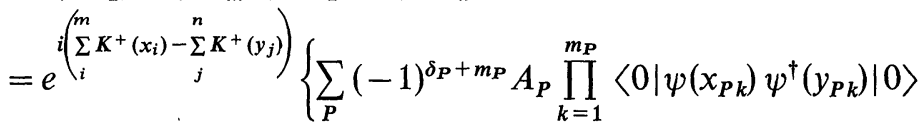

$$
\begin{aligned}
& \left.\left.\cdot g(Q) e^{i\left(\sum_{l}^{m} \Omega\left(x_{Q l}\right)-\sum_{r}^{n_{Q}} \Omega\left(y_{Q r}\right)\right.}\right): \psi\left(x_{Q 1}\right) \ldots \psi\left(x_{Q m_{Q}}\right) \psi^{\dagger}\left(y_{Q 1}\right) \ldots \psi^{\dagger}\left(y_{Q n_{Q}}\right):\right\} \\
& \cdot e^{i\left(\sum_{i}^{m} K^{-}\left(x_{i}\right)-\sum_{j}^{n} K^{-}\left(y_{j}\right)\right)},
\end{aligned}
$$

where the summation is over all sets of pairs

$$
P=\left\{\left(x_{P 1}, y_{P 1}\right),\left(x_{P 2}, y_{P 2}\right), \ldots,\left(x_{P m_{P}}, y_{P m_{P}}\right)\right\}
$$

and $Q$ is the set of all $x_{i}, y_{j}$ not included in $P$. Moreover

$$
\begin{aligned}
A_{P} & \left.=\sum_{R \subset P}(-1)^{m_{P}-m_{R}} e^{i\left(\sum_{i}^{m_{R}} \Omega\left(x_{R i}\right)-\sum_{j} m_{R} \Omega\left(y_{R_{j}}\right)\right)}\right) \\
& =\prod_{i}^{m_{P}}\left(e^{i\left(\Omega\left(x_{P_{i}}\right)-\Omega\left(y_{P_{i}}\right)\right)}-1\right) .
\end{aligned}
$$

As we have seen in the case of the twofold product, the quantity

$$
\left(e^{i\left(\Omega\left(x_{P_{i}}\right)-\Omega\left(y_{P_{i}}\right)\right.}-1\right)\left\langle 0\left|\psi\left(x_{P i}\right) \psi^{\dagger}\left(y_{P i}\right)\right| 0\right\rangle
$$

approaches a direction-independent limit when $x_{P i}-y_{P i} \rightarrow 0$. Hence, when all $\varepsilon_{i}$ tend to zero, $N\left[\phi\left(x_{i}\right) \ldots \phi\left(x_{n}\right) \phi^{\dagger}\left(y_{i} \ldots \phi^{\dagger}\left(y_{n}\right)\right]\right.$ also approaches a limit, namely

$$
\begin{aligned}
& N\left[\prod_{i}^{m} \phi_{\alpha_{i}} \prod_{j}^{n} \phi_{P_{j}}^{\dagger}\right](x) \\
= & e^{i \chi^{+}(x)}\left\{\sum_{P}(-1)^{\delta_{P}+m_{P}} c_{P}(x) d_{Q}(x): \prod_{i}^{m_{Q}} \psi_{\alpha_{Q i}} \prod_{j}^{n_{Q}} \psi_{\beta_{Q j}}^{\dagger}:(x)\right\} e^{i \chi^{-}(x)}
\end{aligned}
$$

where

$$
\begin{aligned}
x^{ \pm}(x) & =(m-n) \alpha J^{ \pm}(x)+\beta\left(\sum_{i}^{m} \gamma_{x_{i}}^{5}-\sum_{j}^{n} \gamma_{y_{j}}^{5}\right) \tilde{J}^{ \pm}(x), \\
c_{P}(x) & =\prod_{i}^{m_{P}} \frac{1}{2 \pi}\left(\gamma^{\mu} \gamma_{0_{0}}\right)_{\alpha_{i} \beta_{P_{i}}} \partial_{\mu} \Delta(x)\left(\alpha Q+\beta \gamma_{P i}^{5} \tilde{Q}\right), \\
d_{Q}(x) & =\left[g(Q) \exp i\left(\sum_{i}^{m_{Q}} \Omega\left(x_{Q i}\right)-\sum_{j}^{n_{Q}} \Omega\left(y_{Q i}\right)\right)\right]_{x_{i}=y_{j}=x} .
\end{aligned}
$$

The meaning of the summation over $P$ is the same as in Eq. (5.4). 
It should be noted that, due to Fermi statistics, $N\left[(\phi)^{m}\left(\phi^{\dagger}\right)^{n}\right](x)$ vanishes for $m>2$ or $n>2$. However, $N\left[\phi\left(x_{1}\right) \ldots \phi\left(x_{m}\right) \phi^{\dagger}\left(y_{1}\right) \ldots \phi^{\dagger}\left(y_{n}\right)\right]$ does not vanish identically for those values, and the derivatives of sufficiently high order will approach nontrivial limiting fields. In all cases locality and covariance follow by the same arguments presented in the case of the twofold normal product, and requirement $(D)$ of Section III is readily verified.

\section{b) Expansion of Products}

Let us now turn to the question of expanding products $\phi\left(x_{1}\right) \ldots \phi\left(x_{m}\right)$ - $\phi^{\dagger}\left(y_{1}\right) \ldots \phi^{\dagger}\left(y_{n}\right)$ in terms of normal products. We obtain the desired expression by inverting Eq. (5.3), which we first rewrite using more succinct notation,

$N\left[\phi, \phi^{\dagger}\right]_{X}=\sum_{S}(-1)^{\delta_{S}+m_{S}}\langle S\rangle f(T)^{-1} e^{i K^{+}(S)} \prod\left[\phi, \phi^{\dagger}\right]_{T} e^{i K^{-}(S)}$

with the obvious identifications. Just as in the case of Wick products of free fields, we may invert this expression to obtain

$$
\prod\left[\phi, \phi^{\dagger}\right]_{X}=f(X) \sum_{S}(-1)^{\delta_{S}}\langle S\rangle e^{i K^{+}(S)} N\left[\phi, \phi^{\dagger}\right]_{T} e^{i K^{-}(S)} .
$$

This is the analogue of Eq. (3.1). Clearly the presence of the exponential factors gives the expansion of $\phi\left(x_{1}\right) \ldots \phi\left(x_{m}\right) \phi^{\dagger}\left(y_{1}\right) \ldots \phi^{\dagger}\left(y_{n}\right)$ a rather different character from that of $\psi\left(x_{1}\right) \ldots \psi\left(x_{m}\right) \psi^{\dagger}\left(y_{1}\right) \ldots \psi^{\dagger}\left(y_{n}\right)$. Because $K^{-}(S)$ has a simple commutator with $\Pi\left[\phi, \phi^{\dagger}\right]$ but not with $N\left[\phi, \phi^{\dagger}\right]$, it is perhaps more natural to write (5.7) in the form

$$
\Pi\left[\phi, \phi^{\dagger}\right]_{X}=f(X) \sum_{S}(-1)^{\delta_{S}}\langle S\rangle N\left[\phi, \phi^{\dagger}\right]_{T ; S},
$$

with the inverse relation

$$
\begin{aligned}
N\left[\phi, \phi^{\dagger}\right)_{X ; Y} & =\sum_{S}(-1)^{\delta_{S}+m_{S}}\langle S\rangle f(T)^{-1} e^{i K^{+}(S \cup Y)} \Pi\left[\phi, \phi^{\dagger}\right] e^{i K^{-}(S \cup Y)} \\
& =\sum_{S} h_{S}(X, Y): e^{i K(S \cup Y)}: \Pi\left[\phi, \phi^{\dagger}\right]_{T}
\end{aligned}
$$

where

$$
\begin{aligned}
h_{S}(X, Y)= & (-1)^{\delta_{S}+m_{S}}\langle S\rangle f(T)^{-1} \\
& \cdot \exp (-i)\left\{\sum_{i}^{m_{Q}}\left(\sum_{j}^{m_{T}} F\left(x_{R i}, y_{R i}, x_{T_{j}}\right)-\sum_{k}^{n_{T}} F\left(x_{R i}, y_{R i}, y_{T k}\right)\right)\right\},
\end{aligned}
$$




$$
\begin{aligned}
F(x, y, z)= & {\left[\alpha(\alpha-\sqrt{\pi})+\beta(\beta-\sqrt{\pi}) \gamma_{x}^{5} \gamma_{z}^{5}\right] D^{-}(x-z) } \\
& -\left[\alpha(\alpha-\sqrt{\pi})+\beta(\beta-\sqrt{\pi}) \gamma_{y}^{5} \gamma_{z}^{5}\right] D^{-}(y-z) \\
& +\left[\alpha \sqrt{\pi} \gamma_{x}^{5}+\beta \sqrt{\pi} \gamma_{z}^{5}\right] \tilde{D}^{-}(x-z)-\left[\alpha \sqrt{\pi} \gamma_{y}^{5}+\beta \sqrt{\pi} \gamma_{z}^{5}\right] \tilde{D}^{-}(y-z), \\
& R=S \cup Y, \quad X=S \cup T=\left\{x_{1}, \ldots x_{m} ; y_{1}, \ldots y_{n}\right\} \\
Y= & \left\{x_{Y 1}, \ldots x_{Y m_{Y}} ; y_{Y 1}, \ldots y_{Y_{Y}}\right\}, \quad: e^{i K(R)}:=e^{i K^{+}(R)} e^{i K^{-}(R)}
\end{aligned}
$$

We shall call the $N\left[\phi, \phi^{\dagger}\right]_{X ; Y}$ generalized normal products, since clearly

$$
N\left[\phi, \phi^{\dagger}\right]_{X}=N\left[\phi, \phi^{\dagger}\right]_{X ; \emptyset} .
$$

We see that their introduction has made possible the elimination of the awkward sandwiches between positive- and negative-frequency exponentials. To complete the short-distance analysis, we merely insert the Taylor series expansion of $N\left[\phi, \phi^{\dagger}\right]_{T ; S}$ in Eq. (5.8).

\section{c) Normal Product of Currents}

In order to cast the above results in a form which might possibly be generalized to a larger class of models, it will be necessary to express Eqs. (5.8) and (5.9) in a form which involves only the fields $\phi$ and $\phi^{\dagger}$ and the currents $J_{\mu}$ and $\tilde{J}_{\mu}$, without reference to the auxiliary free fields from which they were constructed. In particular, we must define $: \exp i K(X)$ : without reference to the separation $K=K^{+}+K^{-}$. To accomplish this, we define the normal product of currents

$$
\begin{aligned}
N\left(J\left(x_{1}\right) \ldots J\left(x_{m}\right) \tilde{J}\left(y_{i}\right) \ldots \tilde{J}\left(y_{n}\right)\right] \\
=\sum_{\substack{S \\
S \cup T=X \\
S \cap T=\phi}}(-1)^{\frac{m_{S}+n_{S}}{2}}\left\langle 0\left|J\left(x_{S 1}\right) \ldots J\left(x_{S m_{S}}\right) \tilde{J}\left(y_{S 1}\right) \ldots \tilde{J}\left(y_{S n_{S}}\right)\right| 0\right\rangle \\
\quad \cdot J\left(x_{T 1}\right) \ldots J\left(x_{T m_{T}}\right) \tilde{J}\left(y_{T 1}\right) \ldots \tilde{J}\left(y_{T n_{T}}\right),
\end{aligned}
$$

with the inverse formula

$$
\begin{gathered}
J\left(x_{1}\right) \ldots J\left(x_{m}\right) \tilde{J}\left(y_{1}\right) \ldots \tilde{J}\left(y_{n}\right) \\
\quad \sum_{\substack{S \\
S \cup T=X \\
S \cap T=\phi}}\left\langle 0\left|J\left(x_{S 1}\right) \ldots J\left(x_{S m_{S}}\right) \tilde{J}\left(y_{S 1}\right) \ldots \tilde{J}\left(y_{S n_{S}}\right)\right| 0\right\rangle \\
\quad \cdot N\left[J\left(x_{T 1}\right) \ldots J\left(x_{T m_{T}}\right) \tilde{J}\left(y_{T 1}\right) \ldots \tilde{J}\left(y_{T n_{T}}\right)\right] .
\end{gathered}
$$

In the above expressions the vector indices of $J_{\mu}$ and $\tilde{J}_{\mu}$ have been suppressed. The modifications for a product of $J$ 's and $\tilde{J}^{\mu}$ in in some other order are obvious. From the definitions of Section II it is easy to see that the righthand side of (5.11) is equivalent to a Wick ordering of 


$$
\begin{aligned}
& J_{\mu_{1}}\left(x_{i}\right) \ldots J_{\mu_{m}}\left(x_{m}\right) \tilde{J}_{v_{1}}\left(y_{1}\right) \ldots \tilde{J}_{v_{n}}\left(y_{n}\right) \text { with respect to } \\
& J_{\mu_{i}}^{ \pm}\left(x_{i}\right)=j_{\mu_{i}}^{ \pm}\left(x_{i}\right)-\frac{\alpha}{\sqrt{\pi}} \partial_{\mu_{i}} \Delta^{ \pm}\left(x_{i}\right) Q-\frac{\beta}{\sqrt{\pi}} \partial_{\mu_{i}} \tilde{\Delta}^{ \pm}\left(x_{i}\right) \tilde{Q}, \\
& \tilde{J}_{\mu_{i}}^{ \pm}\left(x_{i}\right)=\tilde{j}_{\mu_{i}}^{ \pm}\left(x_{i}\right)-\frac{\alpha}{\sqrt{\pi}} \partial_{\mu_{i}} \tilde{\Delta}^{ \pm}\left(x_{i}\right) Q-\frac{\beta}{\sqrt{\pi}} \partial_{\mu_{i}} \Delta^{ \pm}\left(x_{i}\right) \tilde{Q} .
\end{aligned}
$$

It is clear that the normal product $N\left[J_{\mu_{1}}\left(x_{1}\right) \ldots \mathrm{J}_{\mu_{m}}\left(x_{m}\right) \tilde{J}_{v_{1}}\left(y_{1}\right) \ldots \tilde{J}_{v_{n}}\left(y_{n}\right)\right]$ is well defined and satisfies the requirements set forth at the beginning of Section III. Since

$$
K^{ \pm}(x)-K^{ \pm}(y)=\sqrt{\pi} \int_{y}^{x} K_{y}^{ \pm}\left(x^{\prime}\right) d x^{\prime \mu},
$$

where $K_{\mu}^{ \pm}=\alpha J_{\mu}^{ \pm}+\beta \gamma^{5} \tilde{J}_{\mu}^{ \pm}$and the line integral is path-independent, we may write, for $X=\left\{x_{1}, x_{2}, \ldots x_{m} ; y_{1}, \ldots y_{m}\right\}$,

$$
\begin{aligned}
: e^{i K(X)}: & =e^{i K^{+}(X)} e^{i K^{-}(X)}=e^{i \sum_{i}^{m}\left(K^{+}\left(x_{i}\right)-K^{+}\left(y_{i}\right)\right)} e^{i \sum_{i}^{m}\left(K^{-}\left(x_{j}\right)-K^{-}\left(y_{j}\right)\right)} \\
& =\left(\exp i \sqrt{\pi} \sum_{i=1}^{m} \int_{y_{i}}^{x_{i}} K_{\mu_{i}}^{+}\left(x_{i}^{\prime}\right) d x_{i}^{\prime \mu_{i}}\right)\left(\exp i \sqrt{\pi} \sum_{j=1}^{m} \int_{y_{1}}^{x_{1}} K_{\mu_{j}}^{-}\left(x_{j}^{\prime}\right) d x_{j}^{\prime \mu_{j}}\right) \\
& =N\left[\exp i \sqrt{\pi} \sum_{i=1}^{m} \int_{y_{i}}^{x_{i}} K_{\mu_{i}}\left(x_{i}^{\prime}\right) d x_{i}^{\prime \mu_{i}}\right] .
\end{aligned}
$$

Hence the $N\left[\phi, \phi^{\dagger}\right]_{X, Y}$ of Eqs. (5.8) and (5.9) may be defined entirely in terms of the fields $\phi, \phi^{\dagger}, J_{\mu}$ and $\tilde{J}_{\mu}$ without reference to the positive- and negative-frequency separations of free fields ${ }^{1}$.

\section{d) Wilson Expansion of the Threefold Product. Reformulation of the Equation of Motion}

We now wish to indicate how the expansion formula (5.7) - or (5.8) may be combined with the expression (4.25) for the current to yield a Wilson expansion of arbitrary products. We shall treat in detail only the threefold product $\phi^{\dagger}\left(x_{1}\right) \phi\left(x_{2}\right) \phi\left(x_{3}\right)$, but the general method should be clear from this special example. As a by-product of the derivation we shall obtain two alternative forms of the equation of motion.

1 The appearance of exponential factors of the type (5.13) is not something new. Similar factors appear in Hagen's [6] definition of the Thirring model current, based on a hypothesis of Schwinger [7]. The normal products introduced above give a mathematically satisfactory definition of Hagen's formally defined operators. 
From (5.7) we may write

$$
\begin{aligned}
& \frac{\phi^{\dagger}\left(x_{1}\right) \phi\left(x_{2}\right) \phi\left(x_{3}\right)}{f\left(x_{1}, x_{2}, x_{3}\right)}=N\left[\phi^{\dagger}\left(x_{1}\right) \phi\left(x_{2}\right) \phi\left(x_{3}\right)\right] \\
& +\frac{\varepsilon_{12}^{\mu} \gamma_{\mu} \gamma^{0}}{2 \pi i \varepsilon_{12}^{2}} e^{-i\left(K^{+}\left(x_{1}\right)-K^{+}\left(x_{2}\right)\right)} \phi\left(x_{3}\right) e^{-i\left(K^{-}\left(x_{1}\right)-K^{-}\left(x_{2}\right)\right)} \\
& \quad-\frac{\varepsilon_{13}^{\mu} \gamma_{\mu} \gamma^{0}}{2 \pi i \varepsilon_{13}^{2}} e^{-i\left(K^{+}\left(x_{1}\right)-K^{+}\left(x_{3}\right)\right)} \phi\left(x_{2}\right) e^{-i\left(K^{-}\left(x_{1}\right)-K^{-}\left(x_{3}\right)\right)} \\
& =N\left[\phi^{\dagger} \phi \phi\right]\left(x_{1}\right)+\frac{\varepsilon_{12}^{\mu} \gamma_{\mu} \gamma^{0}}{2 \pi i \varepsilon_{12}^{2}}\left[\phi\left(x_{3}\right)-i \sqrt{\pi} \varepsilon_{12}^{v}\left(K_{v}\left(x_{1}\right) \phi\left(x_{3}\right)\right]\right. \\
& \left.\quad-\left[K_{v}^{-}\left(x_{1}\right), \phi\left(x_{3}\right)\right]\right)-\frac{\varepsilon_{13}^{\mu} \gamma_{\mu} \gamma^{0}}{2 \pi i \varepsilon_{12}^{2}} \\
& \quad \cdot\left[\phi\left(x_{2}\right)-i \sqrt{\pi} \varepsilon_{13}^{v}\left(K_{v}\left(x_{1}\right) \phi\left(x_{2}\right)-\left[K_{v}^{-}\left(x_{1}\right), \phi\left(x_{2}\right)\right]\right)\right]+O(\varepsilon) .
\end{aligned}
$$

To conform to Wilson's hypothesis, we assume in (5.14) that $x_{i}=x+\varepsilon \xi_{i}$ where $\xi_{i}$ are fixed two-vectors with $\xi_{i}^{2} \neq 0$ (unless $\left.\xi_{i}=0\right)$ and $\left(\xi_{i}-\xi_{j}\right)^{2} \neq 0$. We must now find a way to rewrite the terms involving $K_{v}\left(x_{1}\right) \phi\left(x_{3}\right)$ and $K_{v}\left(x_{1}\right) \phi\left(x_{2}\right)$ as sums of normal products. In particular, we may use (4.22) and (4.24) to write

$$
\begin{aligned}
K_{v}\left(x_{1}\right) \phi\left(x_{3}\right)= & \left(\alpha g_{v e}+\beta \gamma_{1}^{5} \varepsilon_{v e}\right) J^{e}\left(x_{1}\right) \phi\left(x_{3}\right), \\
J_{\mu}\left(x_{1}\right) \phi\left(x_{3}\right)= & \frac{\phi^{\dagger}\left(x_{1}\right) \gamma^{0} \gamma_{\mu} \phi\left(x_{2}\right) \phi\left(x_{3}\right)}{f\left(x_{1}, x_{2}\right)}-\frac{\varepsilon_{12 \mu}}{\pi i \varepsilon_{12}^{2}} \phi\left(x_{3}\right) \\
& +\left(\frac{\alpha}{\sqrt{\pi}} \frac{\varepsilon_{12 \mu} \varepsilon_{12}^{v}}{\varepsilon_{1,2}^{2}}+\frac{\beta}{\sqrt{\pi}} \frac{\tilde{\varepsilon}_{12 \mu} \tilde{\varepsilon}_{12}^{v}}{\tilde{\varepsilon}_{12}^{2}}\right) \\
& \cdot J_{v}\left(x_{1}\right) \phi\left(x_{3}\right)+O\left(\varepsilon_{12}\right) \phi\left(x_{3}\right)
\end{aligned}
$$

where

$$
\tilde{\varepsilon}_{12}^{\varrho}=\varepsilon_{12 \sigma} \varepsilon^{\sigma \varrho} .
$$

From the definition of $F(X)$, we have

$$
\left.\frac{f\left(x_{1}, x_{2}, x_{3}\right)}{f\left(x_{1}, x_{2}\right)}\right|_{\gamma_{1}^{5}=\gamma_{2}^{5}}=\left(\frac{\varepsilon_{23}^{2}}{\varepsilon_{13}^{2}}\right)^{\frac{a+b \gamma_{1}^{5} \gamma_{2}^{5}}{4 \pi}}=1-\frac{a+b \gamma_{1}^{5} \gamma_{3}^{5}}{2 \pi} \frac{\left(\varepsilon_{12} \cdot \varepsilon_{13}\right)}{\varepsilon_{13}^{2}}+O\left(\varepsilon_{12}^{2}\right)
$$


so that, from (5.14) and (5.15),

$$
\begin{aligned}
& J_{\mu}\left(x_{1}\right) \phi\left(x_{3}\right)-\left[J_{\mu}^{-}\left(x_{1}\right), \phi\left(x_{3}\right)\right] \\
& =\lim _{\varepsilon_{12} \rightarrow 0}\left\{N\left[\phi^{\dagger} \gamma^{0} \gamma_{\mu} \phi \phi\right]\left(x_{1}\right)-\frac{\varepsilon_{13}^{\tau} \gamma_{\tau} \gamma_{\mu}}{2 \pi i \varepsilon_{13}^{2}} \phi\left(x_{1}\right)+\left(\frac{g}{2 \pi} \frac{\varepsilon_{13}^{\tau} \varepsilon_{13}^{v}}{\varepsilon_{13}^{2}} \gamma_{\tau} \gamma_{\mu}\right.\right. \\
& \left.\quad+\frac{\beta}{2 \sqrt{\pi}} \gamma^{v} \gamma_{\mu}\right)\left(J_{v}\left(x_{1}\right) \phi\left(x_{3}\right)-\left[J_{v}^{-}\left(x_{1}\right), \phi\left(x_{3}\right)\right]\right)-\left(\delta_{\mu}^{e}-\frac{\alpha}{\sqrt{\pi}} \frac{\varepsilon_{12}^{\mu} \varepsilon_{12}^{o}}{\varepsilon_{12}^{2}}\right. \\
& \left.\quad-\frac{\beta}{\sqrt{\pi}} \frac{\tilde{\varepsilon}_{12}^{\mu} \tilde{\varepsilon}_{12}^{o}}{\tilde{\varepsilon}_{12}^{2}}\right)\left[J_{\varrho}^{-}\left(x_{1}\right), \phi\left(x_{3}\right)\right]-\frac{1}{2 \pi i}\left(\frac{a}{\pi} \frac{\varepsilon_{12 \mu} \varepsilon_{12}^{v}}{\varepsilon_{12}^{2}} \frac{\varepsilon_{13 v}}{\varepsilon_{13}^{2}}\right. \\
& \left.\left.\quad+\frac{b}{\pi} \frac{\tilde{\varepsilon}_{12 \mu}}{\varepsilon_{12}^{2}} \tilde{\varepsilon}_{12}^{v} \frac{\tilde{\varepsilon}_{13 v}}{\varepsilon_{13}^{2}} \gamma^{5}\right) \phi\left(x_{2}\right)\right\}+O\left(\varepsilon_{13}\right) \\
& =N\left[\phi^{\dagger} \gamma^{0} \gamma_{\mu} \phi \phi\right]\left(x_{1}\right)-\frac{1}{2 \pi}\left[\frac{1}{i} \partial_{\mu} \phi\left(x_{1}\right)-g\left(J_{\mu}\left(x_{1}\right) \phi\left(x_{3}\right)-\left[J_{\mu}^{-}\left(x_{1}\right), \phi\left(x_{3}\right)\right]\right)\right] \\
& \quad+\frac{\beta}{2 \sqrt{\pi}}\left(\gamma^{v} \gamma_{\mu}\right)\left(J_{v}\left(x_{1}\right) \phi\left(x_{3}\right)-\left[J_{v}^{-}\left(x_{1}\right), \phi\left(x_{3}\right)\right]\right) .
\end{aligned}
$$

Thus, for $g \neq 0$,

$$
\begin{aligned}
J_{\mu}\left(x_{1}\right) \phi\left(x_{3}\right) & -\left[J_{\mu}^{-}\left(x_{1}\right), \phi\left(x_{3}\right)\right] \\
= & \left(1-\frac{\alpha+\beta}{2 \sqrt{\pi}}\right)^{-1}\left\{N\left[\left(\phi^{\dagger} \gamma^{0} \gamma_{\mu} \phi\right) \phi\right]\left(x_{1}\right)-\frac{1}{2 \pi i} \partial_{\mu} \phi\left(x_{1}\right)\right. \\
& \left.-\frac{\beta}{2 g i \sqrt{\pi}} \gamma_{\mu} \gamma^{v} \delta_{\nu} \phi\left(x_{1}\right)\right\}+O\left(\varepsilon_{13}\right) .
\end{aligned}
$$

The last step in (5.16) was made using the identity (derived from the equation of motion)

$$
\begin{aligned}
& \gamma_{\mu} \gamma_{\tau} \partial_{\nu} \phi\left(x_{1}\right)-i g\left(J_{\mu}\left(x_{1}\right) \gamma_{\nu} \gamma_{\tau} \phi\left(x_{3}\right)-\left[J_{\mu}^{-}\left(x_{1}\right), \gamma_{\nu} \gamma_{\tau} \phi\left(x_{3}\right)\right]\right) \\
& =\left(g_{\mu \nu}-\varepsilon_{\mu \nu} \gamma^{5}\right)\left(\partial_{\tau} \phi\left(x_{1}\right)-i g\left(J_{\tau}\left(x_{1}\right) \phi\left(x_{3}\right)-\left[J_{\tau}^{-}\left(x_{1}\right), \phi\left(x_{3}\right)\right]\right)+O\left(\varepsilon_{13}\right)\right.
\end{aligned}
$$

We may now substitute our result in (5.14) to obtain the Wilson expansion

$$
\begin{aligned}
\frac{\phi^{\dagger}\left(x_{1}\right) \phi\left(x_{2}\right) \phi\left(x_{3}\right)}{f\left(x_{1}, x_{2}, x_{3}\right)}= & a\left(\varepsilon_{12}, \varepsilon_{13}\right) N\left[\phi^{\dagger} \phi \phi\right]\left(x_{1}\right)+b\left(\varepsilon_{12}, \varepsilon_{13}\right) \phi\left(x_{1}\right) \\
& +c^{v}\left(\varepsilon_{12}, \varepsilon_{13}\right) \partial_{v} \phi\left(x_{1}\right)+O(\varepsilon) .
\end{aligned}
$$


As a final item, we use Eq. (5.16) to reformulate the equation of motion in two ways:

$$
\begin{aligned}
\frac{1}{i} \gamma^{\mu} \partial_{\mu} \phi(x)=g N\left[\left(\phi^{\dagger} \gamma^{0} \gamma_{\mu} \phi\right) \gamma^{\mu} \phi\right](x), \\
\frac{1}{i} \gamma^{\mu} \partial_{\mu} \phi(x)=\lim _{\epsilon \rightarrow 0} g \gamma^{\mu}\left(\delta_{\mu}^{v}-\frac{\alpha}{\alpha-\sqrt{\pi}} \frac{\varepsilon_{12 \mu} \varepsilon_{12}^{v}}{\varepsilon_{12}^{2}}-\frac{\beta}{\beta-\sqrt{\pi}} \frac{\tilde{\varepsilon}_{12 \mu} \tilde{\varepsilon}_{12}^{v}}{\tilde{\varepsilon}_{12}^{2}}\right) \\
\cdot\left\{\frac{\phi^{\dagger}\left(x_{1}\right) \gamma^{0} \gamma_{\nu} \phi\left(x_{2}\right) \phi\left(x_{3}\right)}{f\left(x_{1}, x_{2}, x_{3}\right)}-\left(\frac{\varepsilon_{12 \nu}}{\pi i \varepsilon_{12}^{2}}-\frac{\varepsilon_{13}^{\tau} \gamma_{\tau} \gamma_{v}}{2 \pi i \varepsilon_{13}^{2}}\right)\right. \\
\left.\cdot \phi\left(x_{3}\right)-\frac{\varepsilon_{13}^{\tau} \gamma_{\tau} \gamma_{\nu} \varepsilon_{12}^{o}}{2 \pi i \varepsilon_{13}^{2}} \partial_{\varrho} \phi(x)\right\}
\end{aligned}
$$

where

$$
x_{i}=x+\varepsilon \xi_{i}, \quad \xi_{i}^{2}=0 \Rightarrow \xi_{i}=0, \quad\left(\xi_{i}-\xi_{j}\right)^{2} \neq 0, \quad i \neq j .
$$

The first form is completely independent of the choice of limiting procedure, and is thus perhaps the most elegant way to state the equation of motion. The second gives the righthand side as a single, uniform limit of a finite sum of ordinary products, and is thus suitable (in the WilsonBrandt scheme) for constructing a perturbative solution of the model. Zimmermann [8] has verified, to arbitrary order in the coupling constant, a similar equation of motion for the self-interacting ( $\lambda A^{4}$ coupling) scalar field in four-dimensional space-time.

Acknowledgment. The author wishes to thank. J. A. Swieca for helpful discussions.

\section{References}

1. Wick, G. C.: Phys. Rev. 80, 268 (1950).

2. Thirring, W.: Ann. Phys. 3, 91 (1958).

3. Klaiber, B.: Boulder lectures 1967, Lectures in theoretical physics, p. 141. New York: Gordon and Breach 1968.

4. Wilson, K.: On products of quantum field operators at short distances. Unpublished Cornell Report.

5. Brandt, R. A.: Ann. Phys. 44, 221 (1967).

6. Hagen, C. R.: Nuovo Cimento 51 B, 169 (1967).

7. Schwinger, J.: Phys. Rev. Letters 3, 296 (1959).

8. Zimmermann, W.: Commun. Math. Phys. 6, 161 (1967).

J. H. Lowenstein

Departamento de Física

Universidade de São Paulo

São Paulo, Brasil 